\title{
The instability of the boundary layer over a disk rotating in an enforced axial flow
}

\author{
Z. Hussain, \\ Department of Mathematics, \\ University of Leicester, \\ LE1 7RH, UK.
}

\author{
S. J. Garrett, \\ Department of Mathematics, \\ University of Leicester, \\ LE1 7RH, UK.
}

\author{
S. O. Stephen, \\ School of Mathematics, \\ University of Birmingham, \\ B15 2TT, UK.
}

October 25, 2011

\begin{abstract}
We consider the convective instability of stationary and traveling modes within the boundary layer over a disk rotating in a uniform axial flow. Complementary numerical and high Reynolds number asymptotic analyses are presented. Stationary and traveling modes of type I (crossflow) and type II (streamline curvature) are found to exist within the boundary layer at all axial flow rates considered. For low to moderate axial flows, slowly-traveling type I modes are found to be the most amplified and quickly-traveling type II modes are found to have the lower critical Reynolds numbers. However, nearstationary type I modes are expected to be selected due to a balance being struck between onset and amplification. Axial flow is seen to stabilize the boundary layer by increasing the critical Reynolds numbers and reducing amplification rates of both modes. However, the relative importance of type II modes increases with axial flow and they are therefore expected to dominate for sufficiently high rates. The application to chemical vapour deposition (CVD) reactors is considered.
\end{abstract}

\section{Introduction}

This paper extends the work of [1] and [2] by considering the effect of a uniform axial flow on the type I and II modes of convective instability within the boundary-layer flow over a rotating disk. This paper is part of a series by the present authors which consider the 
convective instability of the boundary-layer flow over a family of rotating cones (including the disk as the limiting half angle) both in and out of axial flow. This series commenced with an investigation into a family of cones rotating in an otherwise still fluid [3] using both numerical and asymptotic methods, as is done here. The mathematical formulation of the rotating-disk problem in axial flow is necessarily different to that for the rotating cone and this motivates separate publication of the preliminary cone investigation in [4].

The transition of the boundary layer on the surface of rotating disks and cones has been the subject of a number of experimental investigations. These were motivated by the desire to understand transition to turbulence of crossflow vortices, present in three-dimensional boundary layers. Although we are unaware of experimental investigations explicitly into the vortex dynamics within boundary-layer flows over rotating disks placed in axial flow, equivalent experimental investigations on broad cones (half-angle $>40^{\circ}$ ) placed in axial flow are extensive. Early experimental work on such cones was limited to measurements of the transitional Reynolds numbers [5-8]. Following these, detailed flow visualizations and hot-wire measurements by [9-11] demonstrated the existence of co-rotating spiral vortices in the non-turbulent part of the boundary layer, which are fixed on the cone for all rotation and axial flow rates. These vortices are identical to those observed on the disk rotating in still fluid $[12,13]$ and on the rotating sphere [14]. Such vortices are known to arise from type I (crossflow) and type II (streamline curvture) modes of instability. More recently, the experimental investigations of Corke and co-workers [15-17] on disks rotating in otherwise still fluid have shown that traveling modes can be important in the transition process over smooth, clean disks.

Our interest in the rotating-disk boundary layer arises from the desire for completeness in the series of papers on the family of rotating cones and also from industrial applications in the electrochemical industry, in particular. Here flows arising from rotating disks are present in types of chemical vapour deposition (CVD) reactors used for depositing thin films of optical and electrical materials on substrates. Such reactors operate by forcing a carrier 
gas (containing the reactive molecules) onto the substrate held within a disk-like support placed horizontally in the flow. The set-up is contained within a closed reactor and the support is rotated to compensate for any non-uniformity of the incident flow. The gas flow can be considered as a uniform axial flow incident on a rotating disk and it is desirable that the flow close to the substrate be laminar and free from instability to ensure uniform deposition. Although a large amount of literature exists on the theoretical and experimental study of the reactor-flow parameters (see [18-20] and references contained therein), these are concerned with the changes in the laminar-flow profiles that can be achieved and the affect these have on deposition growth rates; no stability analyses exist for the flows in question. We pay particular attention to [18] which considers a simple laminar-flow model that is closely related to the basic flow used in the stability investigation presented here. Although the temperature and compressibility of the carrier gas are important in the physical process of deposition, and are indeed considered in [18] and all following laminar-flow investigations, the incompressible study presented in this paper can be considered as a preliminary investigation into how relevant instability analyses are to the design of CVD processes.

This paper is related to the theoretical investigations of Garrett \& Peake [21], recently revisited by the current authors [4], which consider the absolute instability of the boundarylayer flows over a family of cones rotating within imposed axial flows. There the rotating disk is considered as a special case of cone with half-angle $90^{\circ}$. As can be seen in those papers, the onset of absolute instability is sensitive to the imposed axial flow rate and the location of onset is significantly delayed with increased flow. Absolute instability is not considered further here.

The current paper begins with a description of the mathematical formulation of the problem in $\S 2$ and outlines the asymptotic stability analyses in $\S 3$. We subsequently proceed to study the neutral curves for stationary modes of instability using complementary asymptotic and numerical methods in $§ 4$. The asymptotic analysis for stationary modes is presented in more detail in [22], while the numerical analysis is used to study the linear growth rates 
within the unstable regime. Traveling modes are subsequently studied in $§ 5$ using two distinct formulations. The first is in the rotating frame of reference and presents neutral curves and growth rates for disturbances traveling at fixed frequencies, in an approach that is consistent with, for example, Turkyilmazoglu and Gajjar's work [23-25]. Both asymptotic and numerical approaches are used. The asymptotic approach models traveling modes in axial flow for the first time, and as such builds on the analyses in Hussain's Ph.D. thesis [22] (still fluid) and [23-25] (traveling modes). Meanwhile, the second formulation is in the inertial (stationary) frame of reference and considers disturbances traveling at fixed phase speeds with respect to the disk surface. This is related to Garrett's recent numerical studies of the rotating disk, cone and sphere boundary layers [26-28] where disturbances traveling at around $75 \%$ of each body's surface are found to be most amplified. This is consistent with Kobayashi \& Arai's [29] experimental observation of slow vortices over rotating spheres under particular conditions. In the analysis of stationary modes, identical neutral curves arise in the two frames of reference. Furthermore, in the analysis of traveling modes, the two formulations lead to neutral curves which must be compared at specific values of the local Reynolds number. Following this approach, we observe excellent agreement for a range of traveling modes in axial flow.

\section{Formulation}

The asymptotic and numerical investigations presented in this paper use slightly different formulations. We give a summary of the formulation used in the asymptotic investigation here and mention the amendments relevant to the formulation of the numerical investigations at relevant points in the text.

Consider a rigid disk of infinite extent rotating about the $z^{*}$-axis which passes through the center of the disk. The radial and azimuthal coordinates are $x^{*}$ and $\theta$, respectively, which rotate with the disk surface. The disk is placed in an incompressible fluid with oncoming 
axial flow aligned parallel to the $z^{*}$-axis at upstream infinity. At the edge of the boundary layer the dimensional surface velocity distribution along the disk is given by the well-known potential-flow solution $U_{0}^{*}\left(x^{*}\right)=C^{*} x^{*}$, (see $[30,31]$ for example). Here $C^{*}$ is a scale factor determined by the free-stream axial flow incident on the disk. Asterisks indicate dimensional quantities. A diagram of the formulation can be seen in Figure 1 of [21] when setting the half-angle to $\psi=90^{\circ}$.

The non-dimensionalization of the asymptotic study follows that detailed in [3] when $\psi=90^{\circ}$, leading to the Reynolds number

$$
R=\frac{\Omega^{*} l^{* 2}}{\nu^{*}}
$$

Here $\Omega^{*}$ is the angular speed of rotation of the disk, $l^{*}$ is a characteristic length scale along the disk surface and $\nu^{*}$ is the kinematic viscosity of the fluid. Distances in the $z^{*}$-direction are scaled on the boundary-layer thickness $\delta^{*}=\left(\nu^{*} / \Omega^{*}\right)^{1 / 2}$, leading to the non-dimensional variable $\zeta=z^{*} / \delta^{*}$. We note that the boundary-layer thickness is $O\left(R^{-1 / 2}\right)$. Note that this scaling is different to that used in [18] and this is discussed in $\S 6$.

The basic steady flow over the disk has the form $x U\left(\zeta ; T_{s}\right), x V\left(\zeta ; T_{s}\right)$ and $R^{-1 / 2} W\left(\zeta ; T_{s}\right)$ in the radial, azimuthal and normal directions respectively, and these are determined by the non-dimensional Navier-Stokes and continuity equations at leading order:

$$
\begin{aligned}
W^{\prime}+2 U & =0, \\
W U^{\prime}+U^{2}-(V+1)^{2} & =T_{s}^{2}+U^{\prime \prime}, \\
W V^{\prime}+2 U(V+1) & =V^{\prime \prime},
\end{aligned}
$$

subject to boundary conditions

$$
\begin{array}{r}
U=0, \quad V=0, \quad W=0, \quad \text { on } \zeta=0, \\
U \rightarrow T_{s}, \quad V \rightarrow-1, \quad \text { as } \zeta \rightarrow \infty .
\end{array}
$$



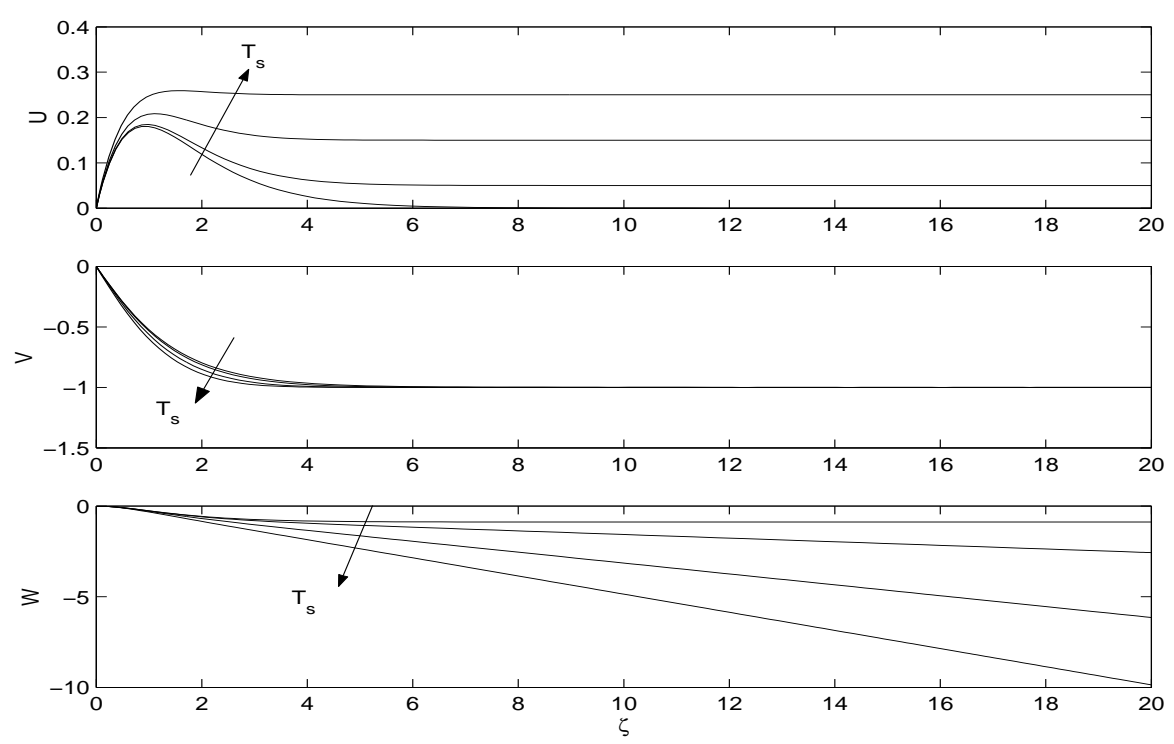

Figure 1: Basic-flow profiles for $T_{s}=0,0.05,0.15$ and 0.25 (arrow indicates direction of increasing $T_{s}$ ).

A prime denotes differentiation with respect to $\zeta$. The parameter $T_{s}$ is the ratio of the local slip velocity at a radial position to the rotational speed of the disk surface at that location:

$$
T_{s}=\frac{C^{*} x^{*}}{\Omega^{*} x^{*}}=\frac{C^{*}}{\Omega^{*}}
$$

We note that in this geometry $T_{s}$ is independent of $x^{*}$ and this simplifies the analysis for the rotating disk; this is a crucial difference between this analysis and that of a rotating cone in axial flow. $T_{s}=0$ represents the disk rotating in otherwise still fluid and we recover the familiar von Kármán equations [32].

Equations (2)-(4), subject to conditions (5), are solved using a fourth-order Runge-Kutta integration method, in conjunction with a two-dimensional Newton-Raphson searching routine to iterate on the outer boundary conditions for different values of $T_{s}$. Figure 1 shows the resulting mean-flow profiles for $T_{s}=0-0.25$. 


\section{$3 \quad$ Linear stability analysis}

We consider type I and type II modes in order to derive estimates of the wavenumber $\gamma_{\delta^{*}}$ and waveangle $\phi$ of the resulting disturbances in the large Reynolds number limit. To derive the linear disturbance equations we introduce small perturbations around the basic flow which lead to governing equations independent of $T_{s}$ and identical to those given by equations (3.1)-(3.4) of [3]. The analysis of each basic-flow profile determined by $T_{s} \neq 0$ then proceeds in a similar way to that given in $[1,3,22]$. For this reason we focus on the quantitative differences that arise in the analysis of $T_{s} \neq 0$ and present only a brief overview of the methods used. The interested reader is referred to $[1,3,22]$ for full details.

\subsection{Inviscid type I modes}

Physically, modes of type I are known to arise from an inflection point in the mean velocity profiles and are inviscid in origin. To analyse these modes the disturbance wavelengths are scaled on the boundary-layer thickness. A small parameter $\epsilon=R^{-\frac{1}{6}}$ is introduced and we define the perturbation velocities and pressure as functions of the wall-normal coordinate $z=\zeta \epsilon^{3}$, in the form

$$
(\tilde{u}, \tilde{v}, \tilde{w}, \tilde{p})=\left(u_{A}(z), v_{A}(z), w_{A}(z), p_{A}(z)\right) \exp \left(\frac{\mathrm{i}}{\epsilon^{3}}\left\{\int^{x} \alpha_{A}(x, \epsilon) \mathrm{d} x+\beta_{A}(\epsilon) \theta-\epsilon \omega_{A} t\right\}\right)
$$

The disturbances associated with these perturbations are neutrally stable and hence $\alpha_{A}$ and $\beta_{A}$ are considered as real quantities. We proceed by expanding the radial and azimuthal wavenumbers as well as the traveling mode frequency in the form

$$
\begin{aligned}
& \alpha_{A}=\alpha_{0}+\epsilon \alpha_{1}+\ldots, \\
& \beta_{A}=\beta_{0}+\epsilon \beta_{1}+\ldots, \\
& \omega_{A}=\omega_{0}+\epsilon \omega_{1}+\ldots,
\end{aligned}
$$


where the perturbation radial velocity in the inviscid layer is expanded as

$$
u_{A}=u_{0}(\zeta)+\epsilon u_{1}(\zeta)+\ldots
$$

Similar expansions are used for $v_{A}, w_{A}$ and $p_{A}$, and the resulting perturbation equations are solved at each order.

Consequently, for the first order eigenmodes, following [1], we observe the existence of two layers; an inviscid layer of thickness $O\left(\epsilon^{3}\right)$ and a viscous layer of thickness $O\left(\epsilon^{4}\right)$ to incorporate the no-slip condition at the wall, which is found by balancing convection and diffusion terms in the disturbance equations. Subsequently, we proceed to match the leadingorder solution in the wall layer, using the wall-normal coordinate $\sigma=\epsilon^{-4} z$, to the first-order solution in the inviscid layer to arrive at the following eignenrelation

$$
\frac{w^{\prime}(0)^{2} \mathrm{Ai}^{\prime}\left(\tau_{0}\right)}{\gamma \int_{\tau_{0}}^{\infty} \operatorname{Ai}(s) d s}=2\left(\alpha_{0} \alpha_{1}+\frac{\beta_{0} \beta_{1}}{x^{2}}\right) I_{1}+\left(\frac{\alpha_{1}}{\beta_{0}}-\frac{\beta_{1} \alpha_{0}}{\beta_{0}^{2}}\right) I_{2}+\omega_{0} \bar{I}_{3}
$$

Here $w$ is the leading-order Rayleigh eigenfunction, normalised such that its wall-gradient $w^{\prime}(0)=1$. Furthermore, $\gamma=\left[\mathrm{i}\left(\alpha_{0} x U^{\prime}(0)+\beta_{0} V^{\prime}(0)\right)\right]^{1 / 3}$ and the integrals $I_{1}, I_{2}$ are defined and calculated numerically in $[1,3,22]$. Meanwhile, the eigenrelation differs from the stationary modes case only in the Airy function terms on the left-hand side, as well as the addition of the third term on the right-hand side, which is observed also by [23] for traveling modes over a rotating disk in still fluid. The wall layer normal coordinate is re-scaled to give $\tau=\gamma \sigma+\tau_{0}$, where $\tau_{0}=-\mathrm{i} \omega_{0} / \gamma^{2}$ and the integral, as defined in [24] for the still fluid problem, is

$$
\bar{I}_{3}=\int_{0}^{\infty} w_{0}^{2}(\theta) \frac{\overline{\bar{U}}^{\prime \prime}(\theta)}{\overline{\bar{U}}^{2}(\theta)} \mathrm{d} \theta
$$

The effective velocity profile $\overline{\bar{U}}=\alpha_{0} x U+\beta_{0} V$ is defined in $[1,3,22]$. In a similar fashion to $I_{2}$ in $[1,3,22], \bar{I}_{3}$ is calculated using numerical integration and the residue theorem due to the singularity present at the location of the inviscid critical layer, where $\overline{\bar{U}}=\overline{\bar{U}}^{\prime \prime}=0$. The 
values of $\bar{I}_{3}$ corresponding to axial flows in the range $T_{s}=0-0.25$ are given in the appendix.

\subsection{Viscous type II modes}

Physically, modes of type II are known to originate from viscous effects close to the disk boundary. To analyse these modes we consider a triple-deck structure to be built on a small parameter, now given by $\epsilon=R^{-\frac{1}{16}}$. The lower, main and upper decks have thicknesses of order $\epsilon^{9}, \epsilon^{8}$ and $\epsilon^{4}$, respectively, and we define inner variables $\xi, \zeta$, and $Z$ to represent $O(1)$ variation within these decks. The wavenumbers are scaled upon a viscous length-scale, so that the velocity and pressure perturbations become

$$
(\tilde{u}, \tilde{v}, \tilde{w}, \tilde{p})=\left(u_{A}(z), v_{A}(z), w_{A}(z), p_{A}(z)\right) \exp \left(\frac{\mathrm{i}}{\epsilon^{4}}\left\{\int^{x} \alpha_{A}(x, \epsilon) \mathrm{d} x+\beta_{A}(\epsilon) \theta-\epsilon^{2} \omega_{A} t\right\}\right)
$$

We proceed by expanding the radial and azimuthal wavenumbers as well as the frequency as

$$
\begin{aligned}
& \alpha_{A}=\alpha_{0}+\epsilon^{2} \alpha_{1}+\epsilon^{3} \alpha_{2}+\ldots, \\
& \beta_{A}=\beta_{0}+\epsilon^{2} \beta_{1}+\epsilon^{3} \beta_{2}+\ldots, \\
& \omega_{A}=\epsilon \omega_{0}+\epsilon^{2} \omega_{1}+\epsilon^{3} \omega_{2}+\ldots
\end{aligned}
$$

noting that the $O(\epsilon)$ terms in $\alpha_{A}, \beta_{A}$ are zero, while $\alpha_{i}, \beta_{i}$ are real quantities (where $i=$ $0,1,2, \ldots)$.

The solution for the first-order problem, which matches with the main deck, follows the form of that found in [25] for the type II modes of a rotating disk in still fluid; the main difference is the presence of the traveling mode frequency leading to an additional term in $\omega_{0}$. This is manifest in the modified eigenrelation

$$
\begin{array}{r}
\gamma_{0}^{2} I_{3}+\frac{\mathrm{i} \gamma_{0} \beta_{0} U^{\prime}(0)}{\left(\beta_{0}-\alpha_{0} x T_{s}\right)^{2}}\left(1+\frac{V^{\prime}(0)^{2}}{U^{\prime}(0)^{2}}\right) I_{4}+\frac{\mathrm{i} \gamma_{0} \omega_{0} \Delta^{\frac{3}{4}}}{\left(\beta_{0}-\alpha_{0} x T_{s}\right)^{2}} I_{5} \\
=\frac{\mathrm{i} \gamma_{0} \Delta^{\frac{1}{2}}}{\left(\beta_{0}-\alpha_{0} x T_{s}\right)^{2}}\left(\alpha_{1} x U^{\prime}(0)+\beta_{1} V^{\prime}(0)\right)
\end{array}
$$


where $\gamma_{0}=\sqrt{\alpha_{0}^{2}+\beta_{0}^{2} / x^{2}}$ is the leading order type II wavenumber and $I_{3}, I_{4}$ are numerical integrals defined and calculated in $[1,3,22]$. The final integral on the left-hand side is also calculated numerically and given by

$$
I_{5}=\frac{\int_{0}^{\infty} \theta^{2} U(0, \theta) \mathrm{d} \theta}{\sqrt{2} U(0,0)}=1.347
$$

where $U(0, \theta)$ is the parabolic cylinder function defined in [33].

As discussed in $[1,3,22]$, we investigate the boundary-layer structure by obtaining leading order solutions in each of the decks. In the upper deck, disturbances decay exponentially, whereas in the main deck we use the no-slip condition to argue that the effective wall shear tends to zero as $\zeta \rightarrow 0$. This was experimentally observed by [13] for $T_{s}=0$ and is numerically verified in $\S 4.2$ for all $T_{s}$. The leading-order radial and azimuthal wavenumbers are therefore chosen such that

$$
\alpha_{0} U^{\prime}\left(0 ; T_{s}\right)+\frac{\beta_{0}}{x} V^{\prime}\left(0 ; T_{s}\right)=0
$$

In the lower deck, the decay of the leading order solution is manifest in terms of the parabolic cylinder function $U\left(0, \sqrt{ } 2 \Delta^{\frac{1}{4}} \xi\right)$ through the balance of viscous and Coriolis forces, where

$$
\begin{aligned}
\Delta & =\frac{\mathrm{i}}{2}\left(\alpha_{0} x U^{\prime \prime}(0)+\beta_{0} V^{\prime \prime}(0)\right) \\
& =-\frac{\mathrm{i} \alpha_{0} x}{2}\left(1+T_{s}^{2}\right)
\end{aligned}
$$

\section{The analysis of stationary modes}

\subsection{Asymptotic analysis in the rotating frame}

For both type I and type II modes, the asymptotic analyses for stationary modes correspond directly to the lack of time-dependent terms in the linearised perturbation equations. 
Hence, the absence of the traveling mode frequency such that $\omega_{A}=0$ leads to the simplification of both the inviscid and viscous stability analyses. For type I modes, there is no need to calculate the integral $\bar{I}_{3}$, whereas for type II modes, the integral $I_{5}$ is not required; both are eliminated from the process of solving eigenrelations $(6,7)$, respectively.

\section{Inviscid type I modes}

As discussed in [3], estimates for $\gamma_{\delta^{*}}$ and $\phi$ for vortices arising from the type I mode are expressed as

$$
\begin{aligned}
\gamma_{\delta^{*}}^{I} & =\left(\alpha_{A}^{2}+\frac{\beta_{A}^{2}}{x^{2}}\right)^{\frac{1}{2}} \\
& =\gamma_{0}+\left(\alpha_{0} \alpha_{1}+\frac{\beta_{0} \beta_{1}}{x^{2}}\right) \epsilon / \gamma_{0}+\ldots \\
& =A_{s}^{I}-B_{s}^{I} R_{L}^{-1 / 3}+\ldots
\end{aligned}
$$

and

$$
\begin{aligned}
\tan \left(\frac{\pi}{2}-\phi^{I}\right) & =\frac{\alpha_{A} x}{\beta_{A}} \\
& =\frac{\alpha_{0} x}{\beta_{0}}+\left(\frac{\alpha_{1}}{\beta_{0}}-\frac{\beta_{1} \alpha_{0}}{\beta_{0}^{2}}\right) x \epsilon+\ldots, \\
& =C_{s}^{I}+D_{s}^{I} R_{L}^{-1 / 3}+\ldots,
\end{aligned}
$$

where the numerical values of $A_{s}^{I}, B_{s}^{I}, C_{s}^{I}$ and $D_{s}^{I}$ are listed in Table 1 . Note that $\gamma_{0}^{2}=\alpha_{0}^{2}+\frac{\beta_{0}^{2}}{x^{2}}$ and $R_{L}=R^{1 / 2} x$ is the Reynolds number based on the boundary-layer thickness (this removes any dependence on the radial location $x$ ). Numerical values for some underlying quantities are given in the appendix for comparison with those calculated by $[1,3,22]$ when $T_{s}=0$. 


\begin{tabular}{c|cccc}
$T_{s}$ & $A_{s}^{I}$ & $B_{s}^{I}$ & $C_{s}^{I}$ & $D_{s}^{I}$ \\
\hline 0.00 & 1.162 & 8.314 & 4.256 & 16.535 \\
0.05 & 1.224 & 8.476 & 3.932 & 12.710 \\
0.10 & 1.294 & 8.817 & 3.561 & 9.449 \\
0.15 & 1.369 & 9.252 & 3.203 & 6.984 \\
0.20 & 1.445 & 9.755 & 2.879 & 5.200 \\
0.25 & 1.522 & 10.335 & 2.596 & 3.918
\end{tabular}

Table 1: Numerical values for the asymptotic expansions of $\gamma_{\delta^{*}}^{I}$ and $\phi^{I}$ in equations (9) and (10), respectively.

\section{Viscous type II modes}

The analysis leads to the following estimates for the properties of the vortices arising from type II instabilities:

$$
\begin{aligned}
\gamma_{\delta^{*}}^{I I} & =\left(\alpha_{A}{ }^{2}+\frac{\beta_{A}^{2}}{x^{2}}\right)^{\frac{1}{2}} R^{-\frac{1}{4}}, \\
& =\frac{\left(1+\frac{V^{\prime}(0)^{2}}{U^{\prime}(0)^{2}}\right)^{\frac{3}{4}}}{\left|1+\frac{V^{\prime}(0) T_{s}}{U^{\prime}(0)}\right|}\left(\frac{U^{\prime}(0) I_{4}}{I_{3}}\right)^{\frac{1}{2}} x^{-\frac{1}{2}} R^{-\frac{1}{4}}+\ldots \\
& =A_{s}^{I I} R_{L}^{-\frac{1}{2}}+\ldots
\end{aligned}
$$

and

$$
\begin{aligned}
\tan \left(\frac{\pi}{2}-\phi^{I I}\right) & =\frac{\alpha_{A} x}{\beta_{A}} \\
& =\frac{\alpha_{0} x}{\beta_{0}}+\epsilon^{2}\left(\frac{\alpha_{1}}{\beta_{0}}-\frac{\beta_{1} \alpha_{0}}{\beta_{0}^{2}}\right) x+\ldots, \\
& =C_{s}^{I I}+D_{s}^{I I} R_{L}^{-\frac{1}{4}}+\ldots,
\end{aligned}
$$

where the numerical values of $A_{s}^{I I}, C_{s}^{I I}$ and $D_{s}^{I I}$ are listed in Table 2. 


\begin{tabular}{c|ccc}
$T_{s}$ & $A_{s}^{I I}$ & $C_{s}^{I I}$ & $D_{s}^{I I}$ \\
\hline 0.00 & 1.225 & 1.207 & 2.312 \\
0.05 & 1.323 & 1.222 & 2.249 \\
0.10 & 1.438 & 1.231 & 2.164 \\
0.15 & 1.571 & 1.232 & 2.060 \\
0.20 & 1.720 & 1.224 & 1.939 \\
0.25 & 1.884 & 1.207 & 1.809
\end{tabular}

Table 2: Numerical values for the asymptotic expansions of $\gamma_{\delta^{*}}^{I I}$ and $\phi^{I I}$ in equations (11) and (12), respectively.

\subsection{Numerical analysis in the inertial frame}

The numerical study uses the length scale provided by the boundary-layer thickness in all spatial scalings and we consider the disk to be rotating in a fixed frame of reference. This formulation is consistent with that discussed in the relevant sections of $[3,4,21,26-28]$ and permits control of the speed at which vortices rotate with respect to the disk within the analysis [34].

The mean flow is found by solving equations equivalent to (2)-(4) but with amendments due to the different frame of reference. The governing disturbance equations are then formed by perturbing the mean flow with quantities

$$
(\hat{u}, \hat{v}, \hat{w}, \hat{p})=(u(\zeta), v(\zeta), w(\zeta), p(\zeta)) \exp \left(\mathrm{i}\left(\alpha x+\beta R_{L} \theta-\gamma t\right)\right)
$$

The quantity $\alpha$ is complex and $\beta$ and $\gamma$ are real, as required by the spatial analysis used here. It is assumed that $\beta$ is $O(1)$. As defined above, $R_{L}$ is the local Reynolds number that arises from using the boundary-layer thickness as the length scale, whereas the non-dimensional normal distance variable $\zeta$ is defined in the asymptotic analysis.

The governing perturbation equations in this formulation are identical to those given as equations (4.2)-(4.7) in [3], and we see that the effect of $T_{s} \neq 0$ is to change the basic flow on which the analysis is performed. The analysis on each basic flow is similar to that described in [3] and interested readers are referred there for further information. 

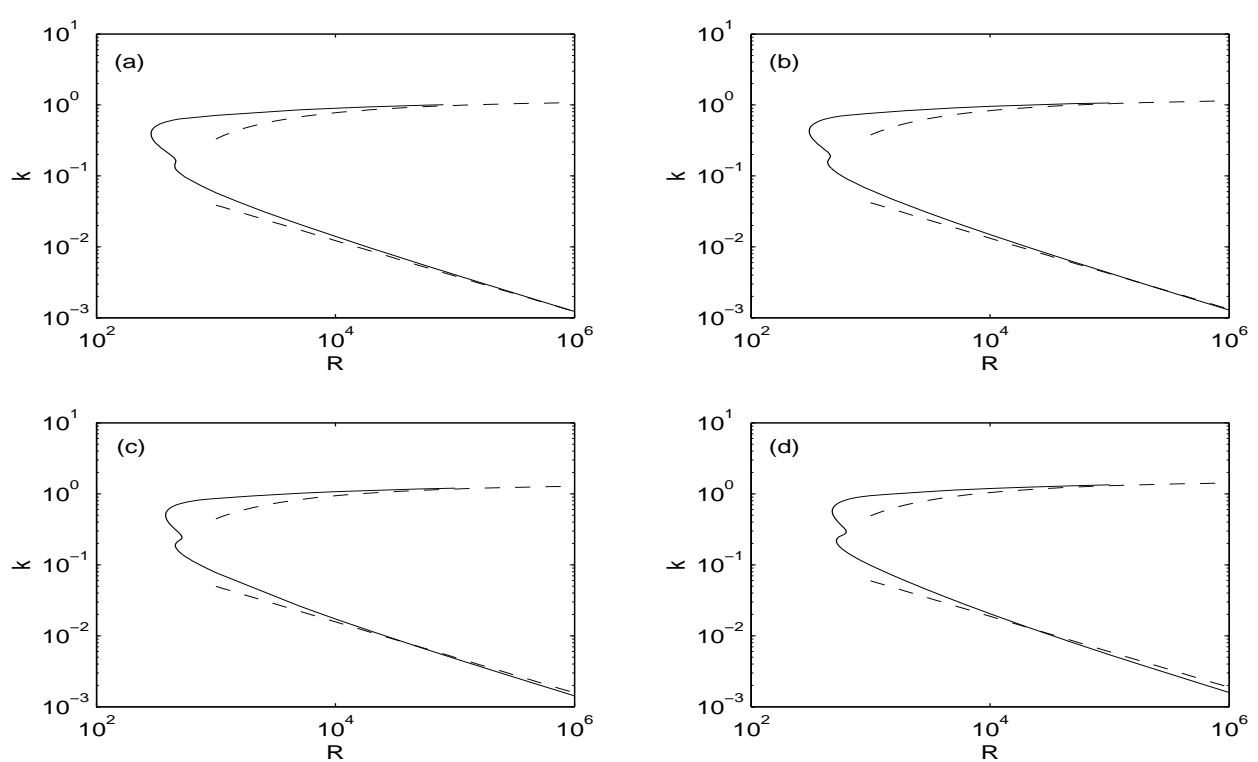

Figure 2: Neutral-stability curves in terms of predicted wavenumber for $T_{s}=0,0.05,0.15$ and 0.25 (a)-(d). Solid line: numerical, dashed line: asymptotic.

\section{Neutral curves}

We begin by explicitly assuming that the vortices rotate with the surface of the disk; this involves fixing the disturbance phase velocity $c=\gamma / \beta=1$. Spatial branches for each $T_{s}$ have been plotted by solving the dispersion relation for complex $\alpha=\alpha_{r}+\mathrm{i} \alpha_{i}$ at fixed $R_{L}$ by marching though values of $\gamma=\beta$. This approach has been denoted method 2 in [26]. At each $T_{s}$, two spatial branches are found to determine the instability of the flow, with behavior similar to those shown in Figure 7 of [3]. These branches arise from crossflow (type I) effects and streamline-curvature (type II) effects. The associated neutral curves (described by $\alpha_{i}=0$ at each $R_{L}$ ) have two distinct lobes with the larger lobe, characterized by higher wavenumbers, due to the type I instability, and the smaller lobe due to the type II instability. The neutral curve calculated for $T_{s}=0$ is identical to that calculated by [2].

Figures 2 and 3 show a comparison between the numerically calculated neutral curves and asymptotic estimates at high Reynolds number, in terms of the wavenumber $k_{\delta}=\sqrt{\alpha^{2}+\beta^{2}}$ and waveangle $\varepsilon=\arctan (\beta / \alpha)$ which are identified with the asymptotic quantities $\gamma_{\delta^{*}}$ and $\phi$, respectively. We see excellent agreement between the numerical and asymptotic predictions 

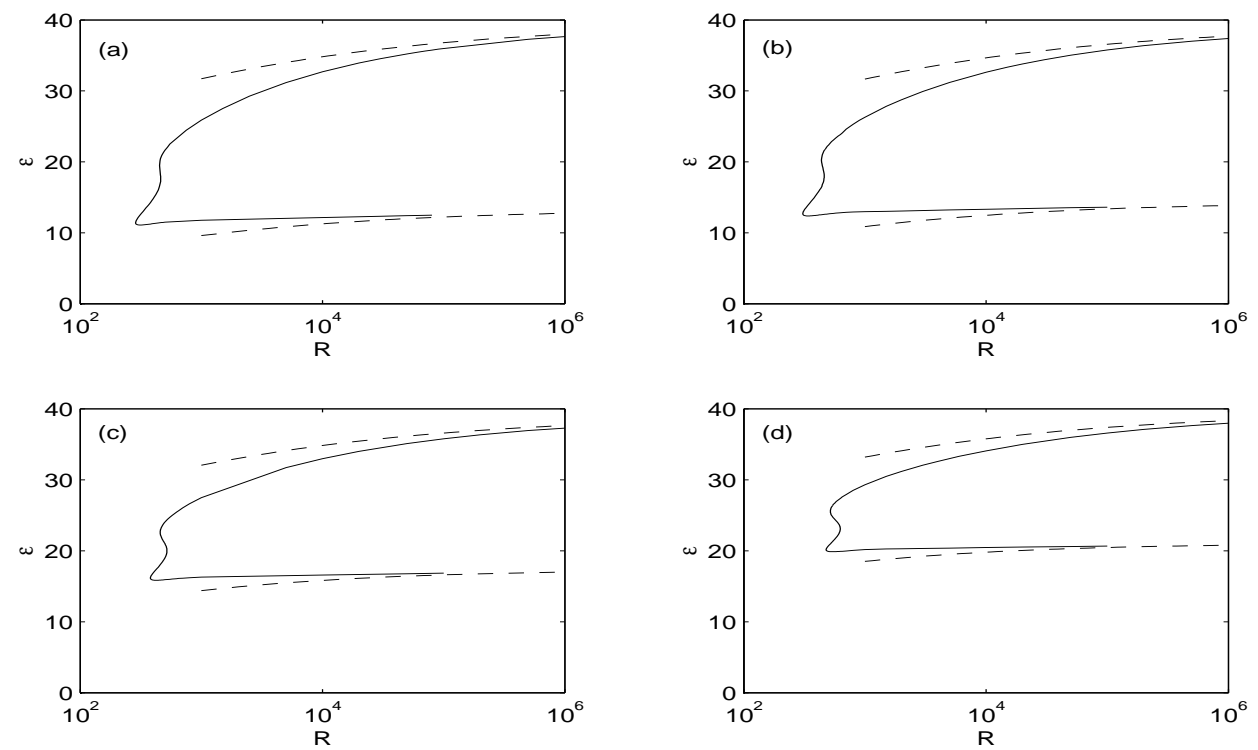

Figure 3: Neutral-stability curves in terms of predicted waveangle for $T_{s}=0,0.05,0.15$ and 0.25 (a)-(d). Solid line: numerical, dashed line: asymptotic.

of wavenumber and waveangle for both modes of instability, even though different frames of reference and formulations have been used.

Figures 2 and 3 show that the type I mode remains the most dangerous (has lower critical $R_{L}$ ) for the values of $T_{s}$ considered. However, as the axial flow rate is increased, the type II mode becomes more important. Figure 4 shows the neutral curves in terms of $\alpha_{r}$ for $T_{s}=0-0.40$, and we see that the type II mode has become the most dangerous by $T_{s}=0.40$. Physically, this behavior is to be expected as increasing the axial flow rate forces fluid towards the disk surface and so any instability is more likely to develop from viscous effects close to the wall location, rather than further up in the boundary layer. The type II modes should therefore become dominant as $T_{s}$ increases. Plots of the effective velocity profile, $Q\left(\zeta ; \Phi, T_{s}\right)=U\left(\zeta ; T_{s}\right) \cos (\Phi)+V\left(\zeta ; T_{s}\right) \sin (\Phi)$, resolved at an angle from the radial direction in the direction of rotation, $\Phi$, show the presence of an inflection point at certain values of $\Phi$ for all values of $T_{s}$ (see Figure 5 for example). Hence the type I mode is expected to exist at all axial flow rates, as is observed in Figure 4.

The asymptotic analysis assumes that the effective wall shear is zero along the lower (type 


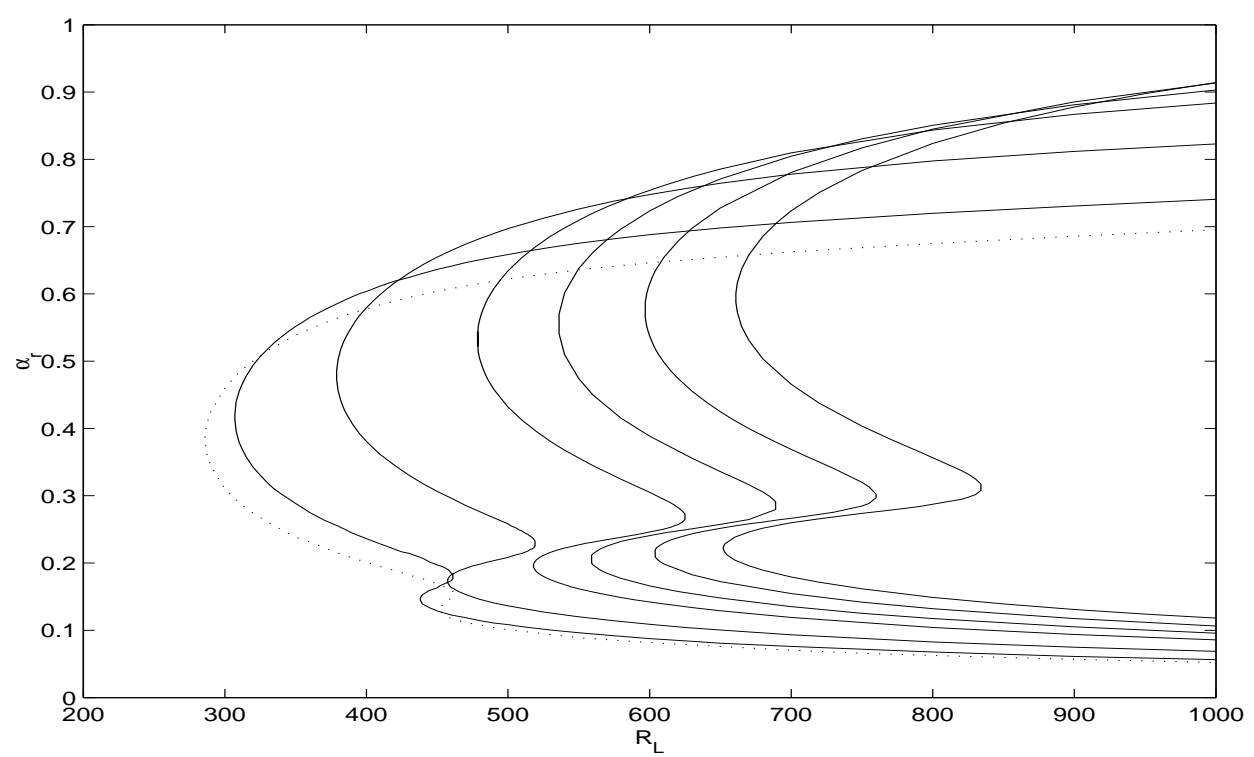

Figure 4: Neutral-stability curves in the $\left(\alpha_{r}, R_{L}\right)$-plane for $T_{s}=0$ (dotted line), 0.05, 0.15, $0.25,0.30,0.35$ and 0.40 (left to right).

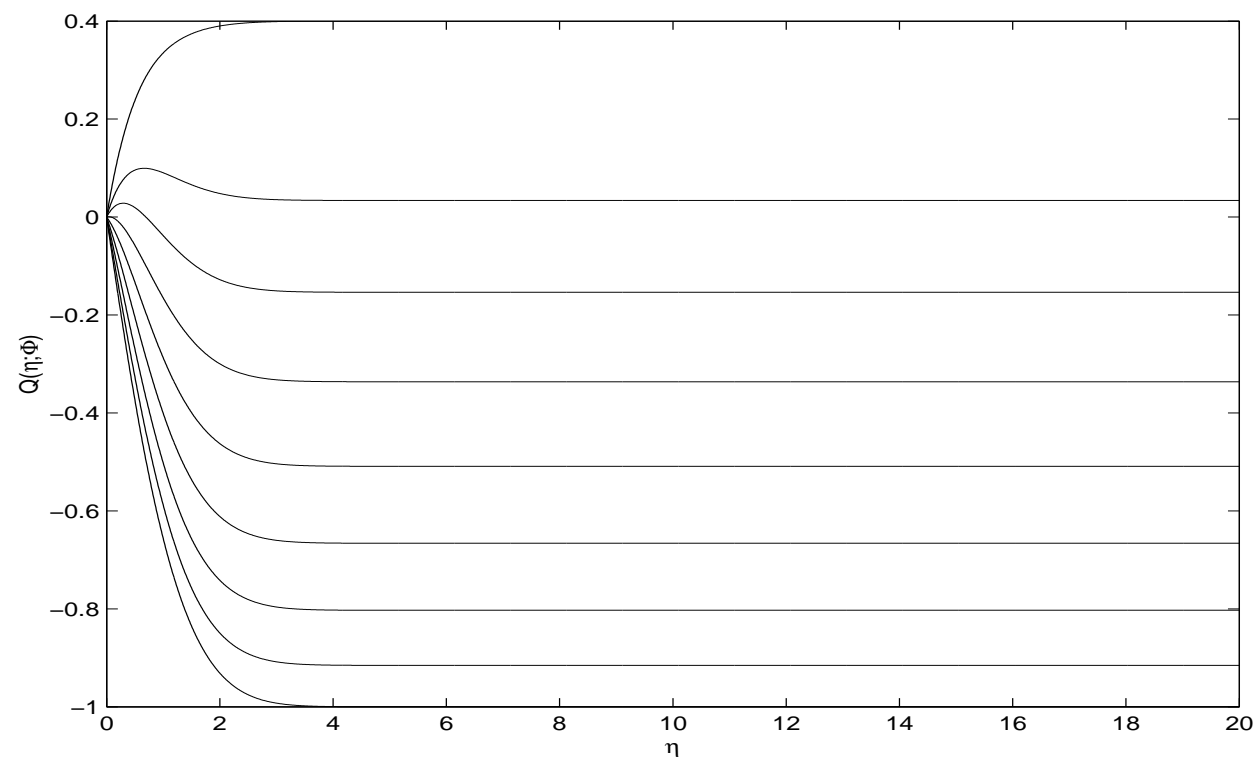

Figure 5: The effective mean velocity profiles (in the rotating frame) for $T_{s}=0.40$ at $\Phi=90^{\circ}-0^{\circ}$ in increments of $10^{\circ}$ (bottom to top). 
II) branch. This assumption is not necessary in the numerical investigation and indeed we are able to justify it using the numerical formulation. The effective wall shear along the lower branch can be calculated from the values of $\alpha U^{\prime}\left(0 ; T_{s}\right)+\beta V^{\prime}\left(0 ; T_{s}\right)$ along the type II branch of the numerical results. This quantity is identified with equation (8) from the asymptotic investigation, and has been investigated for $T_{s}=0-0.40$. We can confirm that its value tends to zero as $R_{L}$ increases and is consistent with the equivalent calculation of [2] for $T_{s}=0$.

\section{Linear amplification rates}

We have so far shown that the critical Reynolds number for the type II mode is reduced relative to the type I mode as the axial flow rate is increased. However, in order to better understand the mode selection process in practice it is necessary to consider the linear growth rates of both modes through the convectively unstable region. In doing this we follow the analysis of [26] that considers linear growth rates within the rotating-disk boundary layer for $T_{s}=0$. That work has since been extended to the boundary-layer flows over the family of rotating cones and spheres by $[27,28]$ with a view to understanding the vortex-speed selection process over smooth bodies (see $\S 5$ ).

In order to compare the effect of incident axial flow, the amplification rates over an equal extent of $R_{L}$ are required. We have chosen to consider a region of extent of 215 in $R_{L}$. This is approximately the extent of the convectively unstable region for $T_{s}=0$ until local absolute instability is encountered at $R_{A} \approx 507$, as determined by [35,36]. This is known to be close to the experimentally observed onset of turbulence for (see [37] for example) and so for comparison purposes can be interpreted as the extent of spiral vortices within the boundary layer. However, recent investigation by [38] suggests that edge effects of the rotating disk may contribute to the linear global instability of the flow, and that infinite rotating disks may remain globally stable despite the existence of local absolute instability. Furthermore, axial flow is known to significantly delay the onset of absolute instability and so potentially 

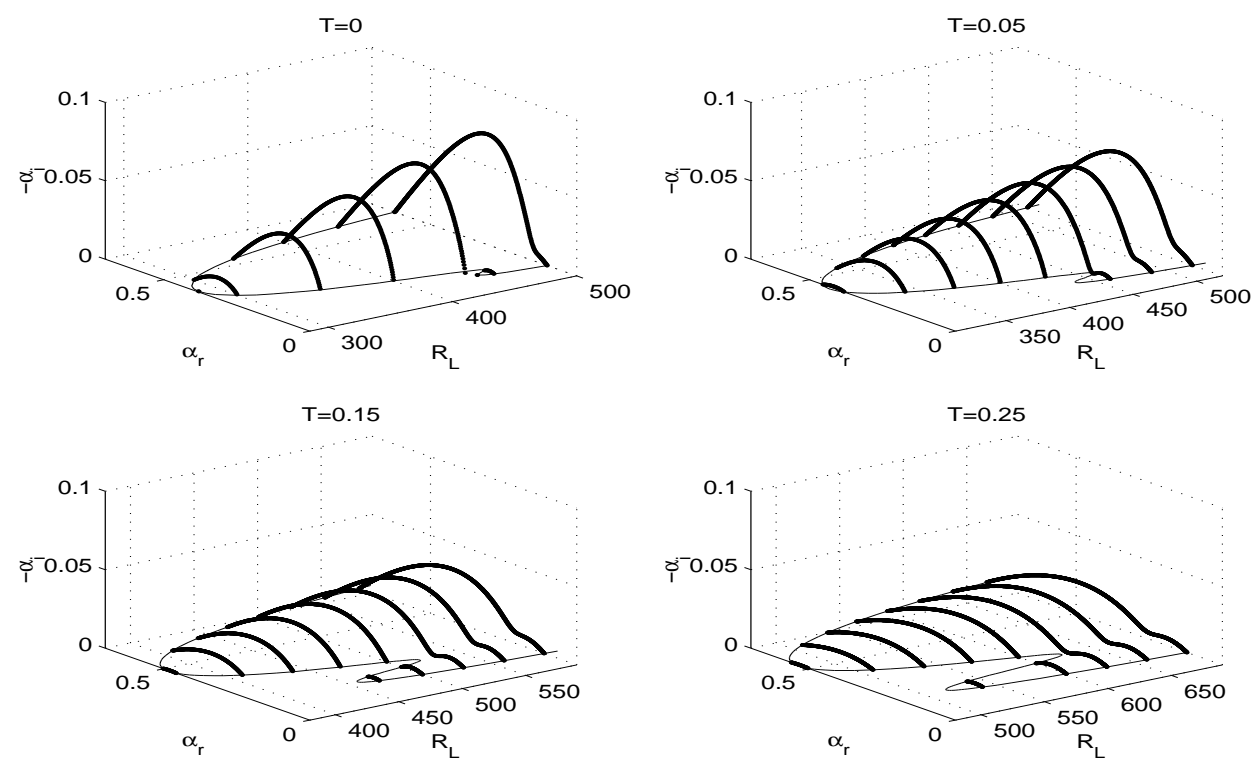

Figure 6: Linear convective growth rates for stationary disturbances at various $T_{s}$ at a fixed extent of 215 in $R_{L}$ into the convectively unstable region.

the onset of turbulence (see $[4,21]$ ). Hence, a range of $R_{L}$ should be considered, and so to facilitate the comparison an extent of 215 is used for all $T_{s}>0$.

Figure 6 shows the amplification rates of stationary modes through a convective unstable region of around 215 in $R_{L}$ from the onset of the type I mode in the range $T_{s}=0-0.25$. We see that the amplification rates are significantly reduced with axial flow and the type II mode becomes increasingly important relative to the type I mode. For sufficiently high axial flow rates the amplification rates of the type II mode is expected to become larger than the type I mode and could be selected in practice. However investigations have showed that this would be at rates far in excess of $T=0.40$. Similar behavior has been found in the rotating-sphere boundary layer in [28].

\section{The analysis of traveling modes}

As discussed in $\S 1$, the recent experimental investigations of Corke and co-workers, for example, have shown that non-stationary modes can be important in the transition process 
over smooth disks. This is particularly relevant in the CVD application where highly polished and clean surfaces are used. It is important to note that this experimental observation is consistent with the theoretical investigations into the boundary-layer flow over a disk rotating in otherwise still fluid due to Turkyilmazoglu and Gajjar [23-25]. There, results are presented which demonstrate that disturbances arising from the type II mode moving at different speeds relative to the disk surface can have considerably lower critical Reynolds numbers than the stationary type I mode.

The stability of traveling disturbances for different values of $T_{s}$ is considered here using two distinct approaches. The first is consistent with the rotating-frame analyses in the literature referred to above, and both numerical and asymptotic analyses are presented within this frame. The second approach is via method 2 within the inertial frame, as discussed in $\S 4.2$. Although it was possible to compare the rotating-frame asymptotic analysis with the inertial-frame numerical analysis in $\S 4.2$, this was only because stationary disturbances were being considered. The approaches in the different frames are inconsistent for traveling modes and direct comparisons of the neutral curves are not possible.

\subsection{Rotating-frame asymptotic analysis}

We now consider the case of traveling modes of frequency $\omega_{A}$ for type I and type II disturbances. In both cases the stability analyses presented are similar to $§ 4.1$ for stationary modes. However, the inclusion of time-dependence in the disturbance equations leads to an additional terms in the eigenrelation for both the inviscid and viscous modes. These distinguish the analyses from those presented in [22] for stationary modes and [23-25] for traveling modes in still fluid.

\section{Inviscid type I modes}

The inviscid traveling mode analysis reveals the non-existence of time-dependent terms in the leading order solution. As a result, we recover the leading-order estimates for the 


\begin{tabular}{c|cc|cc|cc|cc}
$T_{s}$ & $B_{t}^{I}$ & $D_{t}^{I}$ & $B_{t}^{I}$ & $D_{t}^{I}$ & $B_{t}^{I}$ & $D_{t}^{I}$ & $B_{t}^{I}$ & $D_{t}^{I}$ \\
\hline 0.00 & 8.318 & 16.541 & 8.314 & 16.547 & 8.313 & 16.549 & 8.312 & 16.551 \\
0.05 & 8.478 & 12.708 & 8.474 & 12.713 & 8.472 & 12.715 & 8.471 & 12.716 \\
0.10 & 8.843 & 9.500 & 8.839 & 9.503 & 8.837 & 9.505 & 8.836 & 9.506 \\
0.15 & 9.321 & 7.089 & 9.316 & 7.091 & 9.314 & 7.092 & 9.313 & 7.093 \\
0.20 & 9.900 & 5.362 & 9.895 & 5.365 & 9.893 & 5.366 & 9.892 & 5.366 \\
0.25 & 10.596 & 4.134 & 10.591 & 4.136 & 10.588 & 4.137 & 10.587 & 4.137
\end{tabular}

Table 3: First-order numerical values for the asymptotic expansions of $\gamma_{\delta^{*}}^{I}$ and $\phi^{I}$ in equations $(13,14)$, for $x^{-2 / 3} \omega_{0}=-0.0001,-0.000862,0.00017$ and 0.000215 (left to right), which correspond to $\omega=-5,4,7.9$ and 10 , respectively, at $R_{L}=10^{7}$

stationary type I modes, so that for any $\omega_{A} \neq 0$ the leading-order wavenumber $A_{t}^{I}=A_{s}^{I}$ and the leading-order waveangle $C_{t}^{I}=C_{s}^{I}$ for varying axial flow strengths $T_{s}$; in the still fluid case of the rotating disk, the same is observed by [24].

As in $\S 3.1$, we proceed to solve equation (6) for $\omega_{0} \neq 0$ and re-scale in terms of the Reynolds number based on boundary-layer thickness $R_{L}$. Hence, we arrive at the first-order corrections for the type I wavenumber $\gamma_{\delta^{*}}^{I}$ and waveangle $\phi^{I}$, which are given by

$$
\gamma_{\delta^{*}}^{I}=A_{t}^{I}-B_{t}^{I} R_{L}^{-1 / 3}+\ldots
$$

and

$$
\tan \left(\frac{\pi}{2}-\phi^{I}\right)=C_{t}^{I}+D_{t}^{I} R_{L}^{-1 / 3}+\ldots
$$

where the numerical values of $B_{t}^{I}$ and $D_{t}^{I}$ are given in Table 3 for values of $\omega_{A}$ corresponding to numerical traveling-mode frequencies $\omega=-5,4,7.9$ and 10 at $R_{L}=10^{7}$. (see $\S 5.2$ for a definition of $\omega)$.

\section{Viscous type II modes}

In the upper and main decks, the analysis leads to equation (8), due to the requirement of zero effective wall shear. Hence, at leading-order, the traveling mode solution for the 


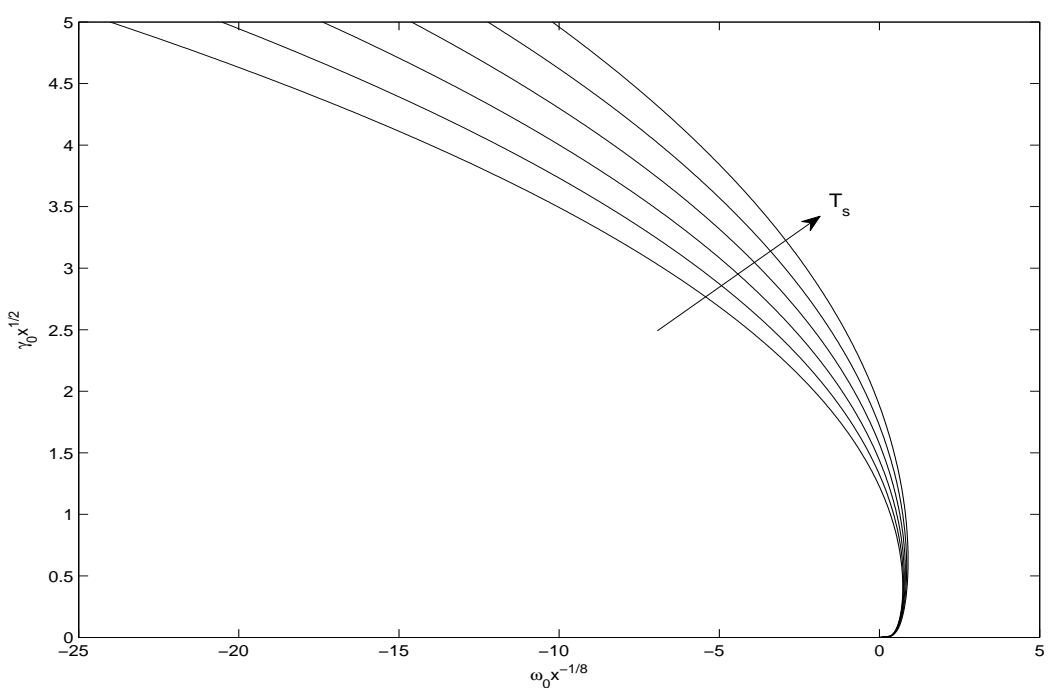

Figure 7: Re-scaled leading-order wavenumber $\bar{\gamma}_{0}$ against the re-scaled frequency $\bar{\omega}_{0}$ shown for increasing axial flow $T_{s}$.

waveangle $\phi^{I I}$ agrees with the stationary mode case, so that $C_{t}^{I I}=C_{s}^{I I}$. Qualitatively, the effects of time-dependent terms in $\phi^{I I}$ do not appear until the first-order problem is considered within the lower deck.

We proceed to solve equation $(7)$ for $\omega_{0} \neq 0$ to obtain estimates for the leading order wavenumber and the first order waveangle. Comparing real and imaginary parts leads to the following eigenrelation

$$
a^{I I} \bar{\omega}_{0}+b^{I I} \bar{\gamma}_{0}^{\frac{1}{4}}-\bar{\gamma}_{0}^{\frac{9}{4}}=0
$$

where $\bar{\omega}_{0}=\omega_{0} x^{-1 / 8}, \overline{\gamma_{0}}=\gamma_{0} x^{1 / 2}$ and the coefficients

$$
\begin{aligned}
a^{I I} & =\frac{\left[\cos \frac{3 \pi}{8}-\sin \frac{3 \pi}{8}\right]}{\left(1+\frac{V^{\prime}(0) T_{s}}{U^{\prime}(0)}\right)^{2}}\left(\frac{V^{\prime}(0)}{U^{\prime}(0)}\right)^{\frac{3}{4}}\left(\frac{1+T_{s}^{2}}{2}\right)^{\frac{3}{4}}\left(1+\frac{V^{\prime}(0)^{2}}{U^{\prime}(0)^{2}}\right)^{\frac{5}{8}} \frac{I_{5}}{I_{3}}, \\
b^{I I} & =\frac{U^{\prime}(0)}{\left(1+\frac{V^{\prime}(0) T_{s}}{U^{\prime}(0)}\right)^{2}}\left(1+\frac{V^{\prime}(0)^{2}}{U^{\prime}(0)^{2}}\right)^{\frac{3}{2}} \frac{I_{4}}{I_{3}} .
\end{aligned}
$$




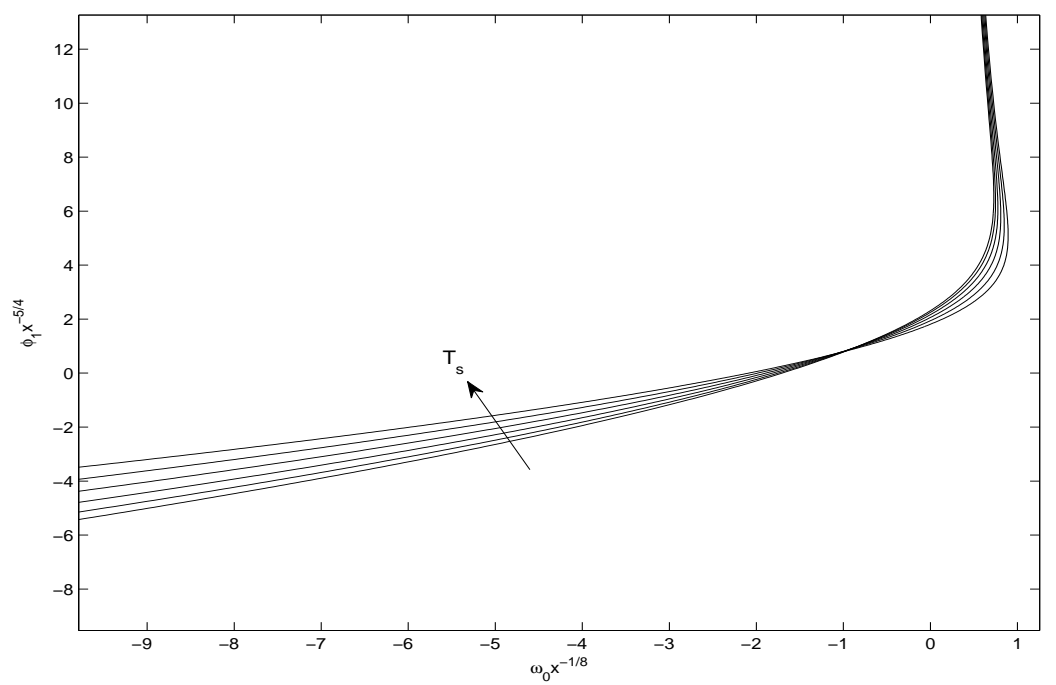

Figure 8: Re-scaled first-order waveangle $\overline{\phi_{1}}$ against the re-scaled frequency $\bar{\omega}_{0}$ shown for increasing axial flow $T_{s}$.

For a given re-scaled frequency $\bar{\omega}_{0}$, the solution to equation (15) is shown in Figure 7, with increasing axial flow having the effect of increasing the re-scaled leading-order wavenumber $\overline{\gamma_{0}}$. Substituting this solution back into equation (7) leads to a corresponding eigenrelation for the first-order waveangle in the form

$$
\bar{\phi}_{1}=b_{1}^{I I}\left(\bar{\gamma}_{0}^{\frac{3}{2}}+a_{1}^{I I} \bar{\gamma}_{0}^{-\frac{3}{4}} \bar{\omega}_{0}\right)
$$

where $\overline{\phi_{1}}=\phi_{1} x^{-5 / 4}$ and the coefficients

$$
\begin{aligned}
& a_{1}^{I I}=\frac{\sin \frac{3 \pi}{8}}{\left(1+\frac{V^{\prime}(0) T_{s}}{U^{\prime}(0)}\right)^{2}}\left(\frac{V^{\prime}(0)}{U^{\prime}(0)}\right)^{\frac{3}{4}}\left(\frac{1+T_{s}^{2}}{2}\right)^{\frac{3}{4}}\left(1+\frac{V^{\prime}(0)^{2}}{U^{\prime}(0)^{2}}\right)^{\frac{5}{8}} \frac{I_{5}}{I_{3}} \\
& b_{1}^{I I}=\frac{2 I_{3}}{\left|U^{\prime}(0) V^{\prime}(0)\right|^{\frac{1}{2}}\left(1+T_{s}^{2}\right)^{\frac{1}{2}}}\left(1+\frac{V^{\prime}(0) T_{s}}{U^{\prime}(0)}\right)^{2}\left(1+\frac{V^{\prime}(0)^{2}}{U^{\prime}(0)^{2}}\right)^{-\frac{1}{4}} .
\end{aligned}
$$

The solution to equation (16) is shown in Figure 8. For small positive $\bar{\omega}_{0}$, increasing axial flow has the effect of reducing the re-scaled first-order waveangle $\bar{\phi}_{1}$, whereas for sufficiently large and negative $\bar{\omega}_{0}, \bar{\phi}_{1}$ is increased. Furthermore, from Figures 7 and 8 , it is clear that for 


\begin{tabular}{c|cc|cc|cc}
$T_{s}$ & $A_{t}^{I I}$ & $D_{t}^{I I}$ & $A_{t}^{I I}$ & $D_{t}^{I I}$ & $A_{t}^{I I, 2}$ & $D_{t}^{I I, 2}$ \\
\hline 0.00 & 1.542 & 1.211 & 0.826 & 3.902 & 0.074 & 16.024 \\
0.05 & 1.657 & 1.201 & 0.908 & 3.729 & 0.072 & 16.465 \\
0.10 & 1.790 & 1.185 & 1.009 & 3.511 & 0.068 & 17.042 \\
0.15 & 1.942 & 1.161 & 1.129 & 3.260 & 0.062 & 17.684 \\
0.20 & 2.110 & 1.127 & 1.266 & 2.993 & 0.056 & 18.356 \\
0.25 & 2.291 & 1.083 & 1.419 & 2.722 & 0.050 & 18.973
\end{tabular}

Table 4: Leading-order numerical values for the asymptotic expansions of $\gamma_{\delta^{*}}^{I I}$ and $\phi^{I I}$ in equations $(17,18)$ for $\bar{\omega}_{0}=-0.667,0.533$ (left to right), which correspond to $\omega=-5,4$, respectively, at $R_{L}=10^{7}$ (the final column is the second branch for $\bar{\omega}_{0}=0.533$ ).

small positive values of $\bar{\omega}_{0}$, a second type II mode exists, which has also been observed in [25] for the type II traveling modes on a rotating disk in still fluid. We subsequently proceed to follow $§ 3.2$, as the solutions to equations $(15,16)$ result in the leading-order estimate for the type II wavenumber $\gamma_{\delta^{*}}^{I I}$ and the first-order correction for the waveangle $\phi^{I I}$ in the form

$$
\gamma_{\delta^{*}}^{I I}=A_{t}^{I I} R_{L}^{-1 / 2}+\ldots
$$

and

$$
\tan \left(\frac{\pi}{2}-\phi^{I I}\right)=C_{t}^{I I}+D_{t}^{I I} R_{L}^{-1 / 4}+\ldots
$$

where the values of $A_{t}^{I I}$ and $D_{t}^{I I}$ are given in Table 4 for values of $\omega_{A}$ corresponding to numerical frequencies $\omega=-5,4$ at $R_{L}=10^{7}$. The second branch is also presented for $\omega=4$. (see $\S 5.2$ for a definition of $\omega$ ).

\subsection{Rotating-frame numerical analysis}

We now present a numerical analysis conducted in the rotating frame of reference. The formulation of the problem is slightly different to that presented in $\S 4.2$ in that Coriolis terms appear at $O\left(1 / R_{L}\right)$ in the perturbation equations and the azimuthal component of the basic flow is modified so as to maintain the non-slip condition. The perturbation equations are not 
stated here, but are identical to those stated as equations (14) - (19) in [35]. To facilitate comparisons with experiments where the frequency spectra of traveling disturbances are studied explicitly (rather than vortex speeds observed), we work in terms of a dimensionless frequency $\omega=\gamma R_{L}$ (see [15-17] for example).

We proceed to calculate neutral curves for fixed $\omega$, which determines $\gamma$ at each location determined by $R_{L}$. Enforcing $\omega>0$ is interpreted as analysing modes that travel at speeds greater than the disk surface. However, the azimuthal wavenumber $\beta$ is now a free parameter and so this analysis differs from the inertial-frame analysis in that we are not fixing the phase speed of disturbances.

This approach is entirely consistent with the theoretical investigation of [23] for $T_{s}=0$, and we use those results to validate the amended version of our code in this frame of reference. Figure 9 shows neutral curves of the wavenumbers, waveangle and number of vortices $n$ for various $\omega$ that are identical to those shown in Figure 5 of [23]. In particular, for $\omega>0$ we see the exaggeration of the lower lobe leading to lower critical Reynolds numbers for the type II mode, and for sufficient $\omega<0$ the lower lobe is removed. We see that a globally minimum critical Reynolds number of $R_{L}=64.47$ occurs for a type II mode when $\omega=7.9$. This critical Reynolds number (and indeed all characteristics calculated for each $\omega$ ) agree with those of Turkyilmazoglu \& Gajjar to the second decimal place. However, as discussed in [3], our previous numerical and asymptotic analyses have failed to reproduce their "kink" in the lower branch at very high $R_{L}$.

That the type II mode is exaggerated for disturbances traveling more quickly than the disk surface is consistent with the results of the inertial-frame analysis presented in $§ 5.4$, although quantitative comparisons of neutral curves are not possible between the two formulations.

Figure 10 demonstrates the effect of increasing the enforced axial flow rate. As with the stationary-mode analysis of $§ 4.2$, axial flow is seen to stabilise the flow to traveling modes of both types. For each $T_{s}$, increasing $\omega$ is again seen to exaggerate the relative importance of the type II mode such that it becomes the most dangerous. The value of $\omega_{\min , T_{s}}$ which 

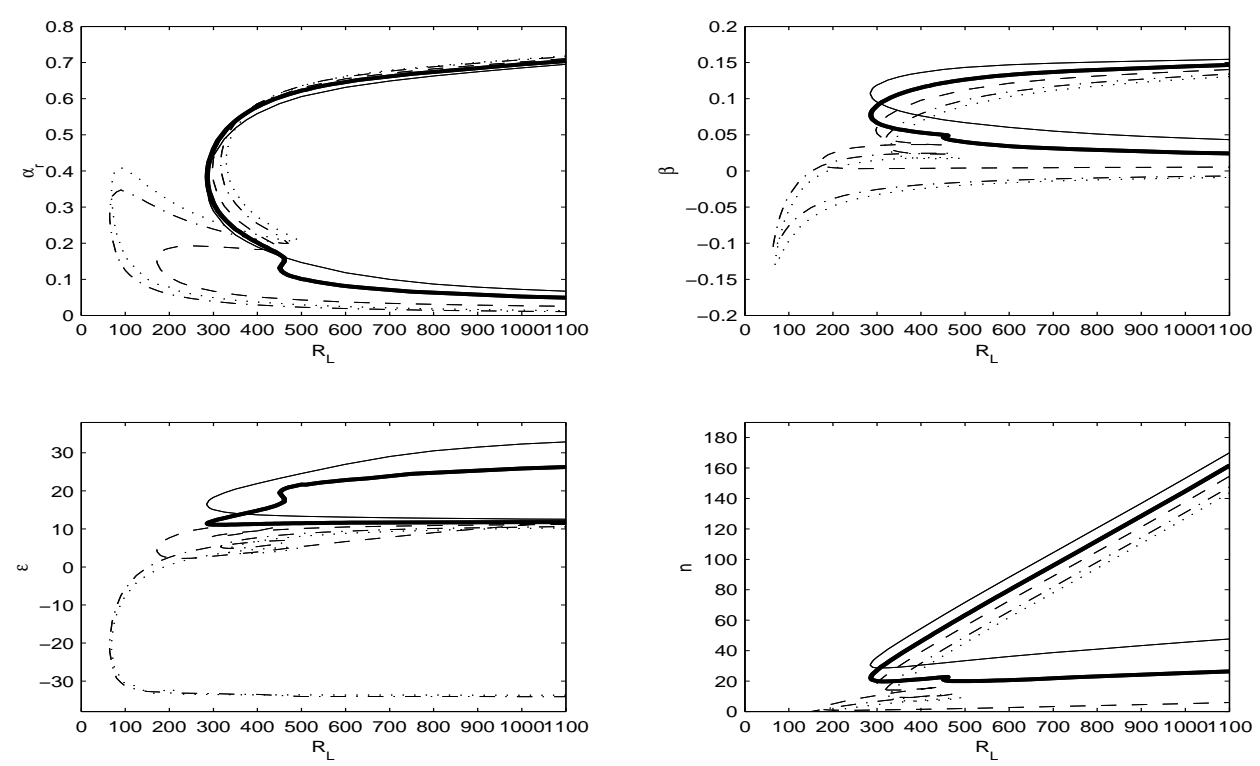

Figure 9: Neutral-stability curves calculated in the rotating frame for $\omega=0$ "-"; $\omega=4$ "--"; $\omega=7.9$ "-."; $\omega=10$ "..."; $\omega=-5 "-"\left(T_{s}=0\right)$.

gives the globally minimum critical Reynolds number is seen to increase slightly with $T_{s}$, in particular $\omega_{\min , 0.05}=8.4, \omega_{\min , 0.15}=10.1$ and $\omega_{\min , 0.25}=11.9$. Calculations of neutral curves for stationary disturbances within the rotating frame $(\omega=0)$ were found to be identical to those calculated in the inertial-frame analysis of $\S 4.2$ for all $T_{s}$.

\section{Linear amplification rates}

As discussed in $\S 4.2$, it is instructive to consider the maximum linear growth rates in each case. We continue by doing this in the rotating frame of reference for $T_{s}=0-0.25$.

Figure 11 demonstrates that a traveling mode with $\omega=-16.5$ is the most amplified in the rotating frame of reference for $T_{s}=0$. This corresponds to a mode traveling more slowly that the disk surface, which is qualitatively consistent with the prediction of slowly traveling vortices in the inertial frame. We also see that the type II mode becomes increasingly important for $\omega>0$, which is again consistent with the results of the inertial-frame analysis. Increasing $\omega$ to around 20 is sufficient for the type II mode to have the greater amplification rate at $T_{s}=0$. However, the critical Reynolds numbers for the onset of both of these modes 

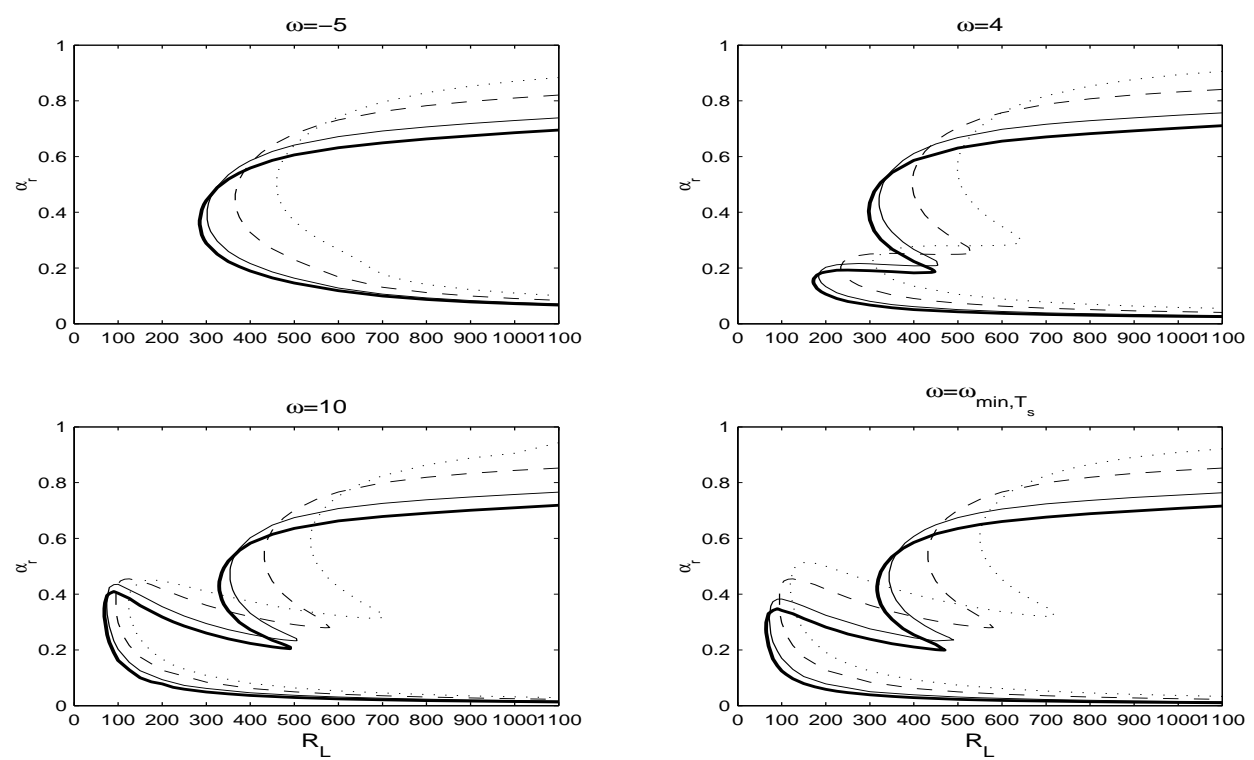

Figure 10: Neutral-stability curves of traveling modes in the rotating frame for $T_{s}=0,0.05$, 0.15 and 0.25 (left to right).

are significantly higher than those that occur for $|\omega|$ closer to zero; this has implications for the mode selection process as discussed in $\S 6$.

Investigation of traveling modes for $T_{s}>0$ have shown that the frequency of the most amplified mode reduces with $T_{s}$. For example, the most amplified mode (as sampled in the latter part of the convectively unstable region prior to the onset of absolute instability) has $\omega=-22.3$ at $T_{s}=0.05$.

\subsection{Comparison between asymptotic and numerical results}

In order to develop consistent comparisons for traveling modes between the asymptotic analysis in $§ 5.1$ and the numerical formulation in $§ 5.2$, we must compare the respective frequencies used in the normal mode expansions of both formulations.

For type I modes, $\omega_{A} \epsilon^{-2}=\omega$. This leads to $\omega_{A} x^{-2 / 3}=R_{L}^{-2 / 3} \omega$, where $R_{L}=R^{1 / 2} x$ and $\epsilon=R^{-1 / 6}$. Hence, at large displacement-thickness Reynolds numbers around $O\left(10^{7}\right)$ and $O(1)$ values of the numerical frequency $\omega$, small corresponding values of $\omega_{A} x^{-2 / 3}$ are required in order to achieve relevant comparisons. For example, for type I modes with 

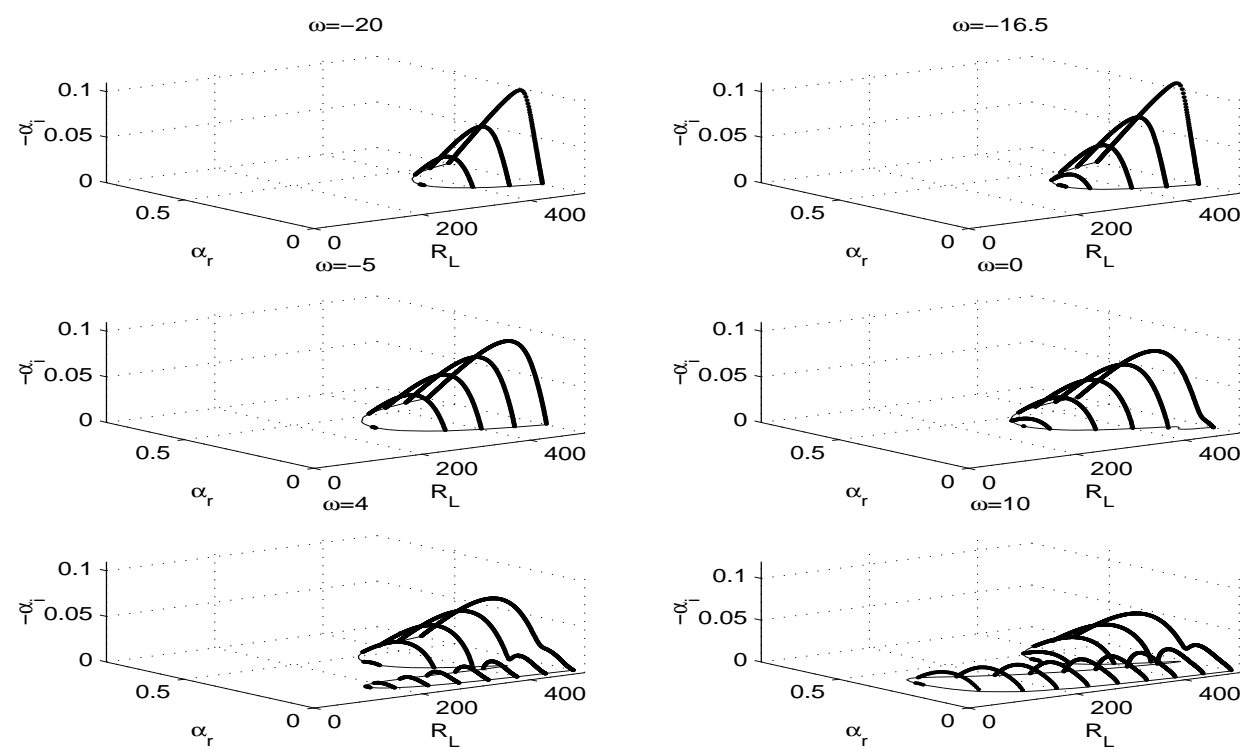

Figure 11: Growth rates for $T_{s}=0$ in the rotating frame for various $\omega$.

numerical frequencies $\omega=-5$ and 4 , we require asymptotic frequencies $\omega_{A} x^{-2 / 3}=-0.0001$ and 0.0000862 , respectively, in order to compare asymptotic and numerical results at the specific displacement-thickness Reynolds number of $R_{L}=10^{7}$.

Similarly, for type II modes, we have $\omega_{A} \epsilon^{-1}=\omega$. As a result, $\omega_{A} x^{-1 / 8}=R_{L}^{-1 / 8} \omega$, where $R_{L}=R^{1 / 2} x$ and for the viscous modes $\epsilon=R^{-1 / 16}$. Therefore, for large Reynolds numbers and $O(1)$ numerical frequencies, again small corresponding values of $\omega_{A} x^{-1 / 8}$ enable suitable comparisons. For example, for type II modes with numerical frequencies $\omega=-5$ and 4 , we require asymptotic frequencies $\omega_{A} x^{-2 / 3}=-0.667$ and 0.533 , respectively, to facilitate comparisons at $R_{L}=10^{7}$. Importantly, from Figures 7 and 8 , we see that there exists no traveling mode solutions for $\omega_{A} x^{-2 / 3}>0.7$, which restricts the range of available comparisons with the numerical results.

Figure 12 shows comparisons applicable at $R_{L}=9 \times 10^{4}$ (the highest value for which the numerical code could obtain traveling mode results for all $\omega$ ), at $T_{s}=0.25$ and $\omega=-5,2$ and 4. We see excellent agreement for the effective wavenumber and waveangle predictions of both mode types (where applicable). Although only these particular cases are shown here, very similar comparisons were found at each $T_{s}$ and $\omega$. The slight discrepancy in the type 

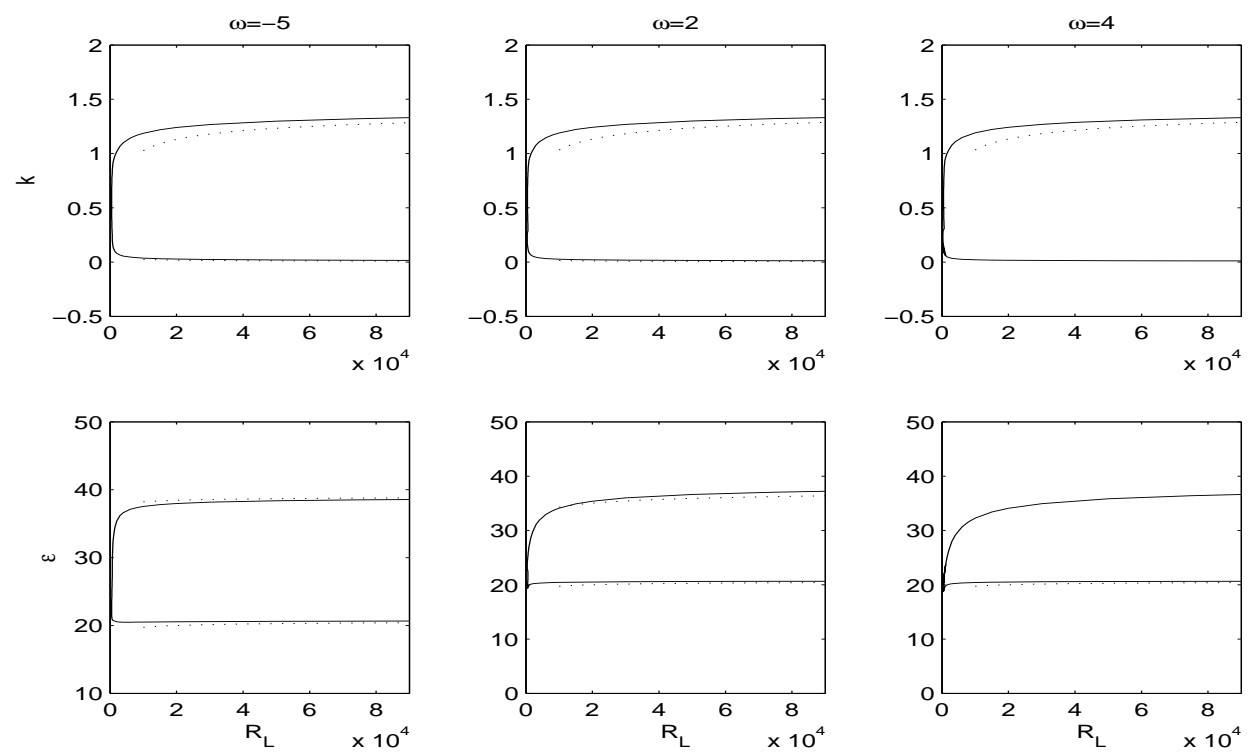

Figure 12: Neutral-stability curves for $T_{s}=0.25$ for traveling modes. Solid lines: numerical, dashed lines: asymptotic.

I effective wavenumber predictions are expected to be a consequence of the relatively low Reynolds number used.

\subsection{Numerical analysis in the inertial frame}

Full neutral curves and amplification rates for vortices traveling at particular speeds relative to the rotating-disk surface can be computed by changing the value of $c$ within the method-2 analysis described in $\S 4.2$, i.e. within the inertial frame.

Disturbance speeds have been considered in the range $c=0.5-20$ at each $T_{s}$ and neutral curves computed. Figures 13-15 present the neutral curves in terms of $\alpha_{r}, n, k_{\delta}$ and $\varepsilon$ for $c=0.8,5$ and 20 together with the $c=1$ results from $\S 4.2$. Note that $c=0.8$ corresponds to disturbances traveling at $80 \%$ of the disk surface speed, and $c=5 \& 20$ corresponds to disturbances traveling at speeds much greater than the disk surface.

In each case we find that the globally critical Reynolds number for the type I mode is given for stationary disturbances, $c=1$. In addition, we find that the lobe arising from the type II mode is sensitive to the disturbance speed. In particular, the type II lobe is quickly 

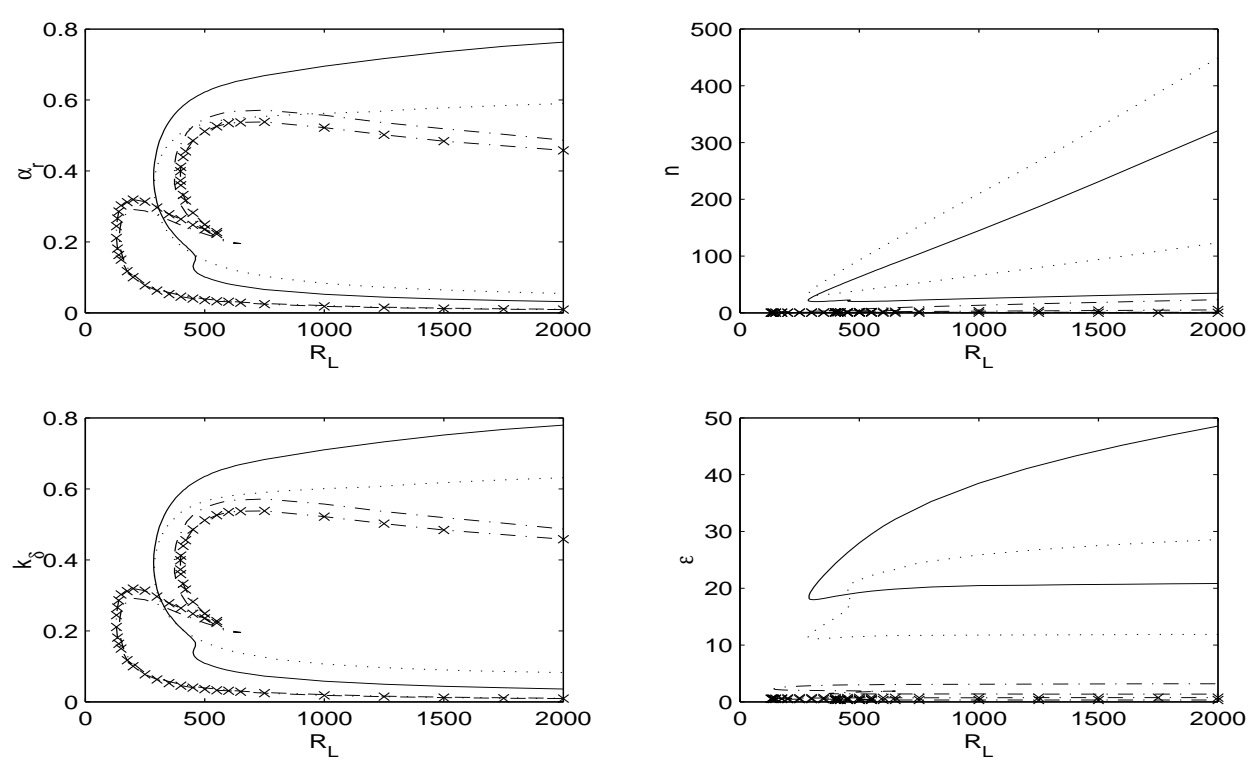

Figure 13: Neutral-stability curves for traveling mode disturbances for $T_{s}=0 ; c=0.8$ "..."; $c=1$ "-"; $c=5$ "-."; $c=20 "-\mathrm{x} "$

eliminated for $c<1$ and exaggerated for $c>1$. In all cases where $c>1$, the type II mode is the more dangerous than in the type I mode in the sense of lowest critical Reynolds number. Imposed axial flow appears to increase the sensitivity of the type II mode to disturbance speed.

The result that traveling type II modes are the most dangerous (in the sense of lowest critical $R_{L}$ ) for all $T_{s}$ is consistent with the previous theoretical results of $\S 5.2$. However, it is important to note that the range of waveangles and vortex numbers predicted to be unstable to traveling modes is extremely narrow, which is in a sense a stabilizing effect. The narrow range of waveangles and vortex numbers appears to be a significant barrier to the selection of quickly traveling modes.

How traveling modes are selected in practice is influenced by the amplification rates within the convectively unstable region. Garrett's previous analysis in [26] has shown that traveling modes with phase speeds of $c=0.75$ are the most amplified in zero axial flow. Investigation of $T_{s}>0$ shows that the phase speed of the most amplified mode increases very slightly with $T_{s}$. For example, the most amplified mode (as sampled in the latter part 

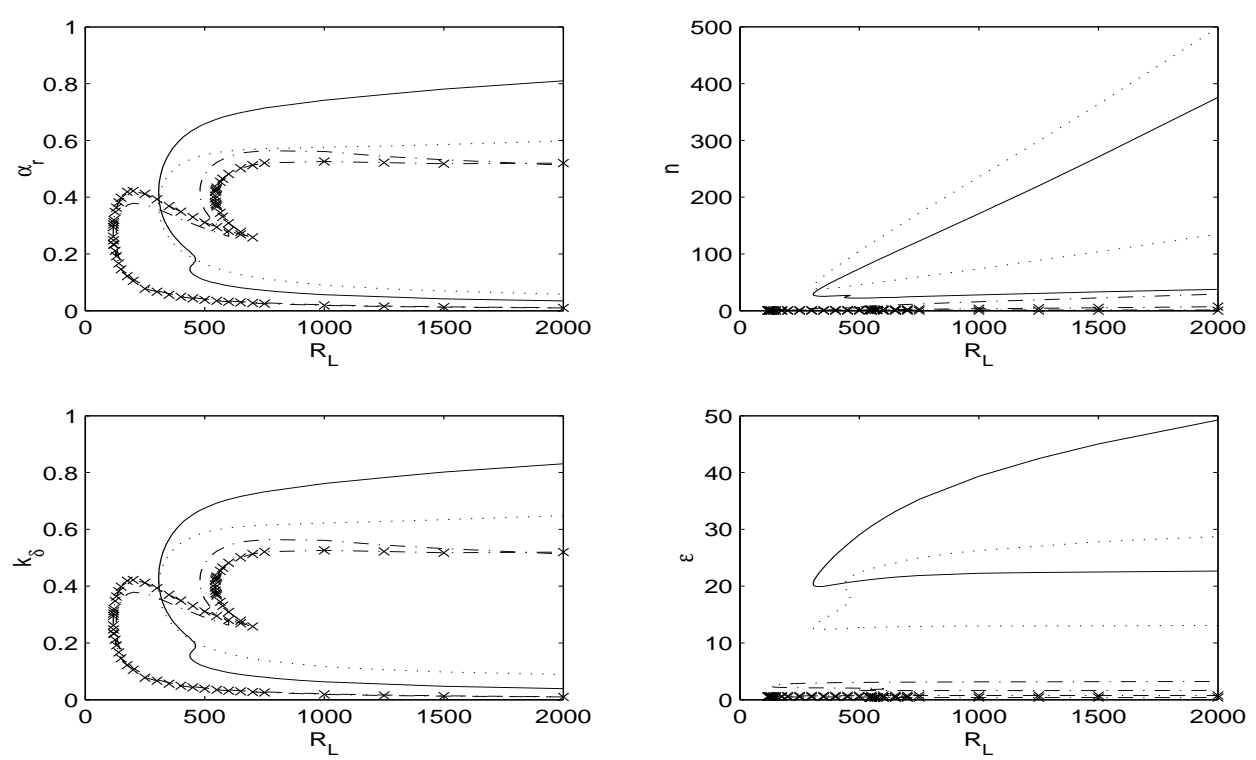

Figure 14: Neutral-stability curves for traveling mode disturbances for $T_{s}=0.05 ; c=$ 0.8 "..."; $c=1$ "-"; $c=5$ "-." ; $c=20$ "-x"
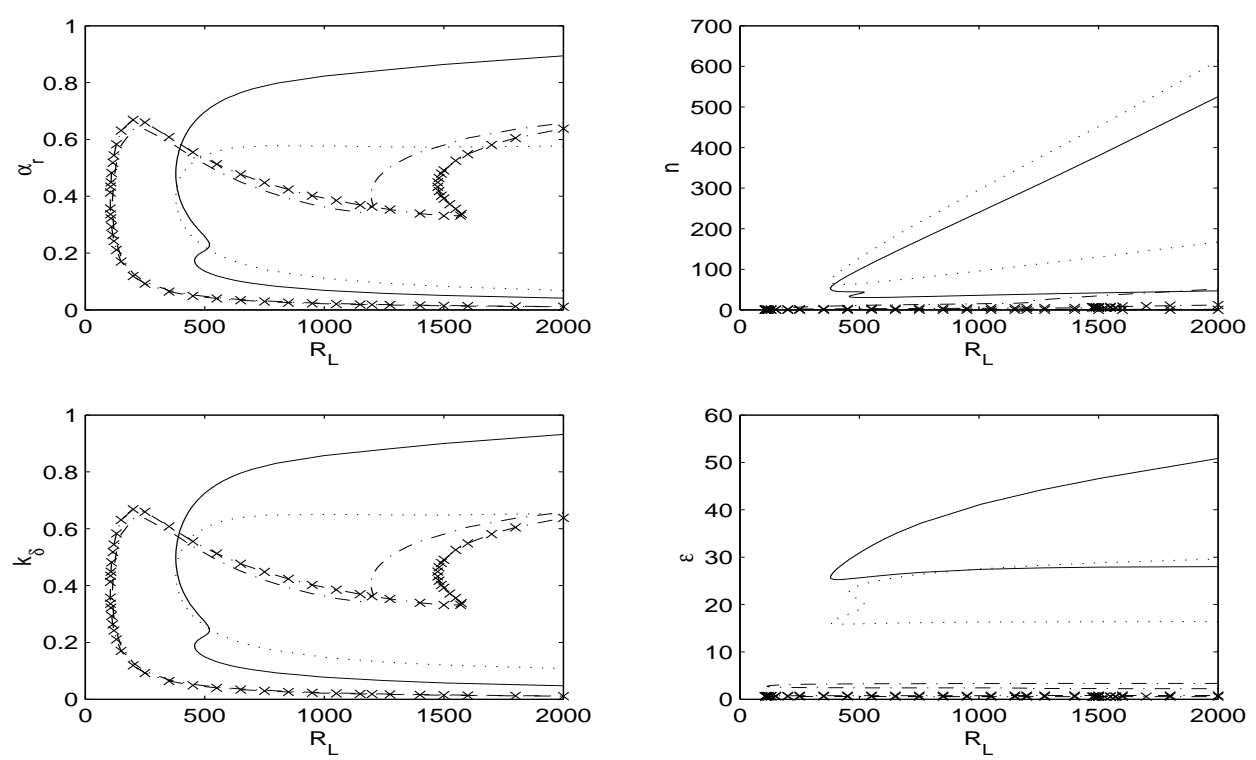

Figure 15: Neutral-stability curves for traveling mode disturbances for $T_{s}=0.15 ; c=$ 0.8 "..."; $c=1$ "-"; $c=5$ "-." ; $c=20$ "-x" 
of the convectively unstable region prior to the onset of absolute instability) remains at $c=0.75$ for $T_{s}=0.05$ and is $c=0.76$ for $T_{s}=0.15$.

\section{Conclusion}

In this paper we have extended the work of $[1,2]$ by considering the theoretical effect of enforcing a uniform axial flow onto the rotating-disk boundary layer. Both stationary and traveling modes have been considered. This investigation is part of a series by the present authors into the convective instability of the boundary layer over a family of rotating cones placed both in and out of axial flow.

The introduction of axial flow necessarily affects the equations governing the basic flow. For the rotating disk, all dependence on the radial position is removed leading to a modified form of the von Kármán ordinary differential equations. This does not happen when axial flow is incident on a rotating cone with $\psi \neq 90^{\circ}$, and the resulting analysis is different. This crucial difference motivates separate publication of the two cases and a preliminary Orr-Sommerfeld analysis of the rotating-cone boundary layer is presented in [4].

\section{Stationary modes}

We began by explicitly studying stationary modes of instability. This is consistent with the existence of surface roughness on the disk (as would naturally occur in many practical applications) which is known to select stationary vortices.

Increasing the axial flow rate is found to stabilize the rotating-disk boundary layer to both type I and type II stationary modes of instability. This is evident from the increasingly narrow range of waveangles predicted to be unstable in both the asymptotic and numerical investigations, and also from the increasing critical Reynolds numbers at the onset of either mode predicted by the numerical investigation. The numerical investigation also demonstrates that both stationary instability modes are evident in the boundary layer for all axial 
flow rates considered, and, although the type I mode is the most dangerous (in the sense of lowest critical Reynolds number) for low rates of axial flow, the type II mode becomes increasingly important with increased $T_{s}$, becoming most dangerous at some critical value around 0.40. Axial flow is seen to reduce the amplification rates of stationary modes, confirming again that it has a stabilising effect. It also acts to increase the importance of the type II mode relative to the type I mode. However, the type I mode is still the most amplified for all $T_{s} \leq 0.40$ considered here.

Physically, these results are to be expected as increasing the axial flow rate forces fluid towards the disk surface and so instabilities are more likely to develop from viscous effects close to the wall location.

The asymptotic analysis necessarily assumes that the effective wall shear is zero along the type II branch, and we have been able to confirm this a posteriori at all $T_{s}$ using the numerical analysis. The asymptotic and numerical investigations have shown consistent predictions of the wavenumber and waveangle of disturbances arising from both type I and II modes, and excellent quantitative agreement has been found in all cases even though the two problems were formulated in different frames of reference.

\section{Traveling modes}

In practical applications where highly polished, smooth disks are used (for example in CVD reactors) non-stationary modes are known to occur in the boundary layer. The stability of traveling modes was considered using two distinct formulations: The first used the rotating frame of reference and allowed control of the disturbance frequency within the boundarylayer flow (consistent with experiments of the type conducted by Corke and co-workers, for example). The second used the inertial frame of reference and allowed control of the vortex speed with respect to the disk surface (consistent with experiments of the type conducted by Kohama \& Arai on the rotating sphere). Although the two formulations are such that direct quantitative comparisons of neutral curves are not possible, the main result that disturbances 
traveling more slowly than the disk surface are the most amplified is found in both cases. Exactly what this means for vortex-speed selection over smooth disks is not clear cut since the type I modes (which dominate the type II modes in all cases considered) were found to have higher critical Reynolds numbers than their stationary counterpart. A balance is therefore to be struck between the near-stationary modes that occur earlier in the unstable region and the more amplified "slow" disturbances that occur later on. It is likely by the point that the "slow" disturbances can arise, the near-stationary modes will have grown sufficiently to remain the most dominant. This is a more subtle process than previously proposed in [26].

As with stationary modes, enforced axial flow was seen to stabilise the boundary layer by increasing the critical Reynolds numbers and also reducing the amplification rates of both modes. It is also seen to increase the relative importance of the type II mode with respect to the type I mode. However, the type I mode is still dominant for all $T_{s}$ considered here. Axial flow is seen to very slightly increase the phase speed of the most amplified traveling mode, even though the frequency of the mode becomes increasingly negative with $T_{s}$.

\section{CVD applications}

The scalings used in this investigation are different to those presented by [18], who investigate the laminar-flow profiles arising from an equivalent (but compressible) model for CVD reactors. Specifically, their boundary-layer thickness is considered to be of order $\sqrt{\nu^{*} /\left(C^{*}+\Omega^{*}\right)}$, which leads to two parameters appearing in their basic-flow equations: $R_{1}=\Omega^{*} /\left(C^{*}+\Omega^{*}\right)$ and $R_{2}=C^{*} /\left(C^{*}+\Omega^{*}\right)$. These parameters can be expressed in terms of our single parameter as $R_{1}=1 /\left(1+T_{s}\right)$ and $R_{2}=T_{s} /\left(1+T_{s}\right)$. Their investigation considers the deposition properties of the laminar flows in the regimes $R_{1}=0, R_{2}=1$ (equivalent to $T_{s} \rightarrow \infty$ ); $R_{1}=0.5, R_{2}=0.5$ (equivalent to $T_{s}=1$ ) and $R_{1}=1, R_{2}=0$ (equivalent to $T_{s}=0$ ). In terms of these parameters, our investigation has $R_{1}=1$ and $R_{2}=T_{s}$ which is consistent with their formulation when $T_{s}<<1$. Our analysis focuses on $T_{s} \leq 0.25$ and we 
therefore conclude that the two analyses are consistent in this range. Our formulation has the advantage that $T_{s}$ does not appear in the perturbation equations and Reynolds number, which greatly simplifies the stability analysis and interpretation of results. However, if we were to consider large axial flow rates (relative to rotation rate) then the scalings used in [18] would be more suitable and compressibility would also need to be considered.

Descriptions within [20] indicate that CVD reactor-flows can reach Reynolds numbers of around $R \sim 10^{4}$, which can be confirmed by noting that a typical length scale within a reactor is around $10 \mathrm{~cm}$ and the substrate can be rotated at rates up to $1500 \mathrm{rpm}$. Our results show that this Reynolds number is well within the unstable region for all values of $T_{s}$ considered. This clearly shows that the stability characteristics of any reactor-flow design should be considered if the laminar flow is to remain in practice.

With regards the simple rotating-disk model of [18], their results indicate that the growth rates of deposition increase with both axial flow rate and rotation rate. This is due to the narrowing of the boundary layer which necessarily increases the concentration of reactive molecules close to the substrate. Our work furthers this conclusion by suggesting that increasing the axial flow rate is to be preferred as it may act to preserve the laminar flow over a greater region of the support disk. However, the impact of compressibility and temperature profiles needs to be considered in the stability analysis to comment further. It is not possible to summarise previous investigations here, although the effect of wall-cooling or heating is expected to be significant.

ZH wishes to acknowledge financial support from the School of Mathematics, University of Birmingham and the EPSRC. SJG wishes to acknowledge study leave granted by the University of Leicester. This work was partially supported by the EPSRC [grant number $\mathrm{EP} / \mathrm{G} 061637 / 1]$ 


\section{A Values from the asymptotic analyses}

Table 5 presents numerical values for some underlying quantities used in the asymptotic analysis. These are given so that comparisons can be made with equivalent quantities calculated by $[1,3]$ when $T_{s}=0$. The definitions of these quantities can be found in $[1,3]$.

\begin{tabular}{c|cccc}
$T_{s}$ & $\bar{\zeta}$ & $I_{1}$ & $I_{2}$ & $\bar{I}_{3}$ \\
\hline 0.00 & 1.458 & 0.0966 & $0.0582+0.0315 \mathrm{i}$ & $-4.4495-1.0023 \mathrm{i}$ \\
0.05 & 1.394 & 0.0868 & $0.0710+0.0408 \mathrm{i}$ & $-4.4166-1.0464 \mathrm{i}$ \\
0.10 & 1.326 & 0.0765 & $0.0902+0.0546 \mathrm{i}$ & $-4.3777-1.0816 \mathrm{i}$ \\
0.15 & 1.260 & 0.0669 & $0.1153+0.0735 \mathrm{i}$ & $-4.3292-1.1069 \mathrm{i}$ \\
0.20 & 1.198 & 0.0584 & $0.1466+0.0982 \mathrm{i}$ & $-4.2703-1.1228 \mathrm{i}$ \\
0.25 & 1.140 & 0.0509 & $0.1843+0.1296 \mathrm{i}$ & $-4.2050-1.1299 \mathrm{i}$
\end{tabular}

Table 5: Numerical values of quantities used in the asymptotic analysis.

\section{References}

[1] HALL, P. 1986 An asymptotic investigation of the stationary modes of instability of the boundary layer on a rotating disk. Proc. R. Soc. Lond. A 406, 93-106.

[2] Malik, M. R. 1986 The neutral curve for stationary disturbances in rotating-disk flow. J. Fluid Mech. 164, 275-287.

[3] Garrett, S. J., Hussain, Z. \& Stephen, S. O. 2009 The crossflow instability of the boundary layer on a rotating cone. J. Fluid Mech. 622, 209-232.

[4] Garrett, S.J., Hussain, Z. \& Stephen, S.O. 2010 Boundary-layer transition on broad cones rotating in an imposed axial flow. AIAA Journal, 48, No. 6, 1184-1194.

[5] Kreith, F., Ellis, D. \& Giesing, J. 1962 An experimental investigation of the flow engendered by a rotating cone. Appl. Sci. Res. A11, 430-440.

[6] Tein, C. L. \& Campbell, D. T. 1963 Heat and mass transfer from rotating cones. J. Fluid Mech. 17, 105-112. 
[7] Kappesser, R., Greif, R. \& Cornet, I. 1973 Mass transfer on rotating cones. Appl. Sci. Res. 28, 442-452.

[8] Salzberg, F. \& Kezios, S. P. 1965 Mass transfer from a rotating cone in axisymmetric flow. J. Heat Transfer 87, 469-476.

[9] Kobayashi, R. \& Izumi, H. 1983 Boundary-layer transition on a rotating cone in still fluid. J. Fluid Mech. 127, 353-364.

[10] Kobayashi, R., Kohama, Y. \& Kurosawa, M. 1983 Boundary-layer transition on a rotating cone in axial flow. J. Fluid Mech. 127, 341-352.

[11] Kohama, Y. 1984 Behaviour of spiral vortices on a rotating cone in axial flow. Acta Mechanica 51, 105-117.

[12] Gregory, N., Stuart, J. T. \& Walker, W. S. 1955 On the stability of threedimensional boundary layers with application to the flow due to a rotating disk. Phil. Trans. R. Soc. Lond. A 248, 155-199.

[13] Fedorov, B. I., Plavnik, G. Z., Prokhorov, I. V. \& Zhukhovitskit, L. G. 1976 Experimental study of hydrodynamics and transfer process of a rotating disk. $J$. Engng Phys. 31, 1448.

[14] Kohama, Y. \& Kobayashi, R. 1983 Boundary-layer transition and the behaviour of spiral vortices on rotating spheres. J. Fluid Mech. 137, 153-164.

[15] Corke, T. C. \& Knasiak, K. F. 1998 Stationary traveling mode cross-flow mode interactions on a rotating disk. J. Fluid Mech. 355, 285-315.

[16] Отhman, H. \& Corke, T. C. 2006 Experimental investigation of absolute instability of a rotating-disk boundary layer. J. Fluid Mech. 565, 63-94. 
[17] Corke, T. C., Matlis, E. H. \& Othman, H. 2007 Transition to turbulence in rotating-disk boundary layers-convective and absolute instabilities $J$ Eng Math 57, 253272.

[18] Chen, K. \& Mortazavi, A. R. 1986 An analytic study of the chemical vapor deposition (CVD) processes in a rotating pedestal reactor. J. Crystal Growth 77, 199-208.

[19] Coltrin, M. E., Kee, R. J. \& Evans, G. H. 1989 A mathematical model of the fluid mechanics and gas-phase chemistry in a rotating disk chemical vapor deposition reactor. J. Electrochem Soc. 136, 819-829.

[20] Vanka, S. P., Luo, G. \& Glumac, N. G. 2004 Parametric effects on thin film growth and uniformity in an atmospheric pressure impinging jet CVD reactor. J. Crystal Growth 267, 22-34.

[21] Garrett, S. J. \& Peake, N. 2007 The absolute instability of the boundary layer on a rotating cone. European. J. Mech. B. 26, 344-353.

[22] Hussain, Z., Stability and transition of three-dimensional rotating boundary layers. PhD Thesis, University of Birmingham, 2010.

[23] Turkyilmazoglu M., \& Gajjar J.S.B. 1998 Absolute and convective instability in the incompressible boundary layer on a rotating disk. CLSCM Report no. CLSCM-1998002, University of Manchester.

[24] Turkyilmazoglu, M. 2006 Convective and absolute instabilities in the incompressible boundary layer on a rotating disk. Hacettepe Journal of Mathematics and Statistics 35, $117-146$.

[25] Turkyilmazoglu, M. 2007 Non-linear and non-stationary modes of the lower branch of the incompressible boundary layer flow due to a rotating-disk. Quart. Appl. Math.65, $43-68$. 
[26] Garretr, S. J. 2010a Vortex-speed selection within the rotating-disk boundary layer. Journal of Algorithms $\& 5$ Computational Technology 4(1), 71-88.

[27] Garrett, S. J. 2010b Linear growth rates of type I \& II convective modes within the rotating-cone boundary layer. Fluid Dyn. Res. 42, 025504.

[28] Garrett, S. J. 2009c Vortex-speed selection within the boundary-layer flow over a rotating sphere placed in an enforced axial flow. European J. Mech. B. 29, 84-92.

[29] Kobayashi, R. \& Arai, T. 1990 Spiral vortex behaviour in transition region and separation of three-dimensional boundary layers on spheres rotating in axial flow. In Lamina-turbulent transition; Proceedings of the IUTAM Symposium, Toulouse, France, Sept. 11-15, 1989 (A91-39901 16-34), eds. D. Arnal \& R. Michel. Berlin and New York, Springer-Verlag, p. 551-557.

[30] Rosenhead, L. 1963 Laminar Boundary Layers; First Edition. Oxford University Press, Oxford.

[31] Evans, H. 1968 Laminer Boundary Layer Theory. Addison-Wesley, Reading, MA.

[32] Kármán, T. von 1921 Uber laminare und turbulente Reiburg. Z. Agnew. Math. Mech. 1, 233-52.

[33] Abramowitz, M. \& Stegun, I. A. eds. (1972) Handbook of Mathematical Functions with Formulas, Graphs, and Mathematical Tables. New York: Dover Publications, ISBN 978-0-486-61272-0.

[34] However, we will revert back to a rotating frame in $§ 5.2$ where the necessary amendments to the formulation are discussed.

[35] Lingwood, R. J. 1995 Absolute instability of the boundary layer on a rotating disk. J. Fluid Mech. 299, 17-33. 
[36] Lingwood, R. J. 1997 Absolute instability of the Ekman layer and related flows. J. Fluid Mech. 331, 405-428.

[37] Lingwood, R. J. 1996 An experimental study of absolute instability of the rotatingdisk boundary-layer flow. J. Fluid Mech. 314, 373-405.

[38] Healey, J. J. 2010 Model for unstable global modes in the rotating-disk boundary layer. J. Fluid Mech. 663, 148-159. 